ANNALES

POLONICI MATHEMATICI

$84.3(2004)$

\title{
A boundary cross theorem for separately holomorphic functions
}

by Peter Pflug and Việt-Anh Nguyên (Oldenburg)

\begin{abstract}
Let $D \subset \mathbb{C}^{n}$ and $G \subset \mathbb{C}^{m}$ be pseudoconvex domains, let $A$ (resp. $B$ ) be an open subset of the boundary $\partial D$ (resp. $\partial G)$ and let $X$ be the 2 -fold cross $((D \cup A) \times B) \cup$ $(A \times(B \cup G))$. Suppose in addition that the domain $D$ (resp. $G$ ) is locally $\mathcal{C}^{2}$ smooth on $A$ (resp. $B$ ). We shall determine the "envelope of holomorphy" $\widehat{X}$ of $X$ in the sense that any function continuous on $X$ and separately holomorphic on $(A \times G) \cup(D \times B)$ extends to a function continuous on $\widehat{X}$ and holomorphic on the interior of $\widehat{X}$. A generalization of this result to $N$-fold crosses is also given.
\end{abstract}

1. Introduction and statement of the main results. In order to recall here the classical cross theorem and to state our results, we need to introduce some notation and terminology. In fact, we keep the main notation from the book by Jarnicki and the first author [6] and from the survey article by Sadullaev [16].

1.1. Plurisubharmonic measures. Let $\Omega \subset \mathbb{C}^{n}$ be an open set. For any function $u$ defined on $\Omega$, let

$$
\widehat{u}(z):= \begin{cases}u(z), & z \in \Omega, \\ \limsup _{w \in \Omega, w \rightarrow z} u(w), & z \in \partial \Omega .\end{cases}
$$

For a set $A \subset \bar{\Omega}$ put

$$
h_{A, \Omega}:=\sup \{u: u \in \mathcal{P S H}(\Omega), u \leq 1 \text { on } \Omega, \widehat{u} \leq 0 \text { on } A\},
$$

where $\mathcal{P} \mathcal{S H}(\Omega)$ denotes the set of all functions plurisubharmonic on $\Omega$.

We first suppose that $\Omega$ is bounded. Then the plurisubharmonic measure of $A$ relative to $\Omega$ is given by

$$
\omega(z, A, \Omega):=\widehat{h_{A, \Omega}^{*}}(z), \quad z \in \Omega \cup A,
$$

where $u^{*}$ denotes the upper semicontinuous regularization of a function $u$.

2000 Mathematics Subject Classification: Primary 32D15, 32D10.

Key words and phrases: cross theorem, holomorphic extension, plurisubharmonic measure. 
From now on let $\Omega$ be an arbitrary (not necessarily bounded) open set and we shall be concerned with the following two cases.

CASE I: $A \subset \Omega$. In this case, we define the plurisubharmonic measure of $A$ relative to $\Omega$ as follows:

$$
\omega(\cdot, A, \Omega):=\lim _{k \rightarrow \infty} h_{A \cap \Omega_{k}, \Omega_{k}}^{*}
$$

where $\left(\Omega_{k}\right)_{k=1}^{\infty}$ is a sequence of relatively compact open sets $\Omega_{k} \subset \Omega_{k+1} \Subset \Omega$ with $\bigcup_{k=1}^{\infty} \Omega_{k}=\Omega$. Observe that the definition is independent of the exhausting sequence $\left(\Omega_{k}\right)_{k=1}^{\infty}$. Moreover, $\omega(\cdot, A, \Omega) \in \mathcal{P} \mathcal{S H}(\Omega)$.

CASE II: $A$ is an open subset of $\partial \Omega$. We suppose in addition that $\Omega$ is locally $\mathcal{C}^{2}$ smooth on $A$ (i.e. for any $\zeta \in A$, there exist an open neighborhood $U=U_{\zeta}$ of $\zeta$ in $\mathbb{C}^{n}$ and a real function $\varrho=\varrho_{\zeta} \in \mathcal{C}^{2}(U)$ such that $\Omega \cap U=$ $\{z \in U: \varrho(z)<0\}$ and $d \varrho(\zeta) \neq 0)$.

In this case, the plurisubharmonic measure of $A$ relative to $\Omega$ is a function on $\Omega \cup A$ given by

$$
\omega(z, A, \Omega):=\lim _{k \rightarrow \infty} \omega\left(z, A_{k}, \Omega_{k}\right), \quad z \in \Omega \cup A,
$$

where the function $\omega\left(\cdot, A_{k}, \Omega_{k}\right)$ is given by $(1.1)$ and $\left(\Omega_{k}\right)_{k=1}^{\infty}$ is a sequence of relatively compact open sets $\Omega_{k} \Subset \mathbb{C}^{n}$ and $\left(A_{k}\right)_{k=1}^{\infty}$ is a sequence of open subsets of $A$ such that

(i) $\Omega_{k} \subset \Omega_{k+1}$ and $\bigcup_{k=1}^{\infty} \Omega_{k}=\Omega$;

(ii) $A_{k} \subset A_{k+1}$ and $A_{k} \subset \partial \Omega \cap \partial \Omega_{k}$ and $\bigcup_{k=1}^{\infty} A_{k}=A$;

(iii) for any $\zeta \in A$ there is an open neighborhood $V=V_{\zeta}$ of $\zeta$ in $\mathbb{C}^{n}$ such that $V \cap \Omega=V \cap \Omega_{k}$ for some $k$.

In Section 3, we shall prove that the definition is independent of the exhausting sequences $\left(\Omega_{k}\right)_{k=1}^{\infty}$ and $\left(A_{k}\right)_{k=1}^{\infty}$ chosen. Moreover, $\left.\omega(\cdot, A, \Omega)\right|_{\Omega} \in$ $\mathcal{P S H}(\Omega)$.

1.2. Cross and separate holomorphicity. Let $N \in \mathbb{N}, N \geq 2$, and let $\emptyset \neq A_{j} \subset \bar{D}_{j} \subset \mathbb{C}^{n_{j}}$, where $D_{j}$ is a domain, $j=1, \ldots, N$. We define an $N$-fold cross $X$, its interior $X^{\mathrm{o}}$ and a new set $A$ as

$$
\begin{aligned}
X & =\mathbb{X}\left(A_{1}, \ldots, A_{N} ; D_{1}, \ldots, D_{N}\right) \\
& :=\bigcup_{j=1}^{N} A_{1} \times \cdots \times A_{j-1} \times\left(D_{j} \cup A_{j}\right) \times A_{j+1} \times \cdots \times A_{N} \\
& \subset \mathbb{C}^{n_{1}+\cdots+n_{N}}=\mathbb{C}^{n}, \\
X^{\mathrm{o}} & =\mathbb{X}^{\mathrm{o}}\left(A_{1}, \ldots, A_{N} ; D_{1}, \ldots, D_{N}\right) \\
& :=\bigcup_{j=1}^{N} A_{1} \times \cdots \times A_{j-1} \times D_{j} \times A_{j+1} \times \cdots \times A_{N}, \\
A & :=A_{1} \times \cdots \times A_{N} .
\end{aligned}
$$


In particular, if $A_{j} \subset D_{j}, j=1, \ldots, N$, then $X=X^{\mathrm{o}}$. Moreover put $\omega(z):=\sum_{j=1}^{N} \omega\left(z_{j}, A_{j}, D_{j}\right), \quad z=\left(z_{1}, \ldots, z_{N}\right) \in\left(D_{1} \cup A_{1}\right) \times \cdots \times\left(D_{N} \cup A_{N}\right)$. For an $N$-fold $\operatorname{cross} X:=\mathbb{X}\left(A_{1}, \ldots, A_{N} ; D_{1}, \ldots, D_{N}\right)$ let

$$
\widehat{X}:=\left\{z=\left(z_{1}, \ldots, z_{N}\right) \in\left(D_{1} \cup A_{1}\right) \times \cdots \times\left(D_{N} \cup A_{N}\right): \omega(z)<1\right\} .
$$

Then the set of all interior points of $\widehat{X}$ is given by

$$
\widehat{X}^{\mathrm{o}}:=\left\{z=\left(z_{1}, \ldots, z_{N}\right) \in D_{1} \times \cdots \times D_{N}: \omega(z)<1\right\} .
$$

We say that a function $f: X \rightarrow \mathbb{C}$ is separately holomorphic on $X^{\circ}$, and write $f \in \mathcal{O}_{\mathrm{s}}\left(X^{\mathrm{o}}\right)$, if for any $j \in\{1, \ldots, N\}$ and $\left(a^{\prime}, a^{\prime \prime}\right) \in\left(A_{1} \times \cdots \times A_{j-1}\right) \times$ $\left(A_{j+1} \times \cdots \times A_{N}\right)$ the function $\left.f\left(a^{\prime}, \cdot, a^{\prime \prime}\right)\right|_{D_{j}}$ is holomorphic on $D_{j}$.

Finally, throughout the paper, the notation $|f|_{M}$ stands for $\sup _{M}|f|$.

1.3. Motivations for our work. We are now able to formulate what we will cite in what follows as the classical cross theorem.

Theorem 1 (Alehyane \& Zeriahi [1]). Let $D_{j} \subset \mathbb{C}^{n_{j}}$ be a pseudoconvex domain and $A_{j} \subset D_{j}$ a locally pluriregular subset, $j=1, \ldots, N$. Then for any $f \in \mathcal{O}_{\mathrm{s}}(X)$, there is a unique $\widehat{f} \in \mathcal{O}(\widehat{X})$ such that $\widehat{f}=f$ on $X$.

There is a long list of papers dealing with this theorem under various assumptions. For a historical discussion, see the survey article [14].

The question naturally arises how the situation changes when the sets $A_{j}$ live on the boundary $\partial D_{j}, j=1, \ldots, N$.

The first results in this direction were obtained by Malgrange-Zerner [17], Komatsu [10] and Drużkowski [2], but only for some special crosses. Recently, Gonchar $[3,4]$ has proved the following remarkable more general theorem.

Theorem 2. Let $D_{j} \subset \mathbb{C}$ be a Jordan domain and let $\emptyset \neq A_{j}$ be an open subset of the boundary $\partial D_{j}, j=1, \ldots, N$. Then for any $f \in \mathcal{C}(X) \cap \mathcal{O}_{\mathrm{s}}\left(X^{\mathrm{o}}\right)$ there is a unique $\widehat{f} \in \mathcal{C}(\widehat{X}) \cap \mathcal{O}\left(\widehat{X}^{\circ}\right)$ such that $\widehat{f}=f$ on $X$. Moreover, if $|f|_{X}<\infty$ then

$$
|\widehat{f}(z)| \leq|f|_{A}^{1-\omega(z)}|f|_{X}^{\omega(z)}, \quad z \in \widehat{X} .
$$

It should be observed that under the hypothesis of Theorem 2 one has $X \subset \widehat{X}$. We remark that Gonchar's original formulation is slightly different. However, his proof still works for this new formulation.

The main purpose of this work is to generalize Gonchar's theorem to higher dimensions. 
1.4. The main result. We are now ready to state the main result.

Main Theorem. Let $D_{j} \subset \mathbb{C}^{n_{j}}$ be a pseudoconvex domain and let $\emptyset \neq A_{j}$ be an open subset of $\partial D_{j}, j=1, \ldots, N$. Suppose in addition that each domain $D_{j}$ is locally $\mathcal{C}^{2}$ smooth on $A_{j}, j=1, \ldots, N$. Then $X \subset \widehat{X}$ and for any $f \in \mathcal{C}(X) \cap \mathcal{O}_{\mathrm{s}}\left(X^{\circ}\right)$, there is a unique $\widehat{f} \in \mathcal{C}(\widehat{X}) \cap \mathcal{O}\left(\widehat{X}^{\circ}\right)$ such that $\widehat{f}=f$ on $X$. Moreover, if $|f|_{X}<\infty$ then

$$
|\widehat{f}(z)| \leq|f|_{A}^{1-\omega(z)}|f|_{X}^{\omega(z)}, \quad z \in \widehat{X} .
$$

We now give a short outline of the proof. The main idea is to combine Gonchar's theorem and the classical cross theorem with the slicing method. More precisely, with each domain $D_{j}$ we shall associate a family of $\mathcal{C}^{2}$ smooth planar domains which are, roughly speaking, the intersection of an open tubular neighborhood of $A_{j}$ in $D_{j} \cup A_{j}$ with the family of complex lines normal to $A_{j}$ parameterized by $A_{j}$. One important property of this family is that the harmonic measures for its domains depend, in some sense, continuously on the parameter of $A_{j}$. Another important property is that there is a relation between the plurisubharmonic measure of $D_{j}$ and the harmonic measure of the domains in the above family. Applying Gonchar's theorem and the slicing method, we shall find an extension $\widehat{f}$ such that $\widehat{f}$ is holomorphic on a subdomain of each domain in this family. The two important properties mentioned above, combined with a variant of the classical cross theorem, will allow us to propagate the holomorphicity from those one-dimensional subdomains to the desired envelope of holomorphy.

The paper is organized as follows.

We begin Section 2 by collecting some background of potential theory and some classical results. Next we establish a uniform estimate for the Poisson kernels which will play an important role in the proof of the Main Theorem.

Based on the results of Section 2, Section 3 develops necessary estimates for the plurisubharmonic measures that will be used later in Section 5 .

Section 4 provides the first step of the proof. More precisely, we will consider the mixed situation where there is at least one $j$ such that $A_{j}$ is inside $D_{j}$. Moreover, we will establish some quantitative versions of the classical cross theorem.

Section 5 establishes the Main Theorem in the case of a 2-fold cross.

The complete proof of the Main Theorem will be given in Section 6 together with some concluding remarks and open questions.

Acknowledgments. The paper was written while the second author was visiting the Carl von Ossietzky Universität Oldenburg being supported by the Alexander von Humboldt Foundation. He wishes to express his gratitude to these organizations. 


\section{Auxiliary results}

2.1. Harmonic measure for a planar domain. We recall some classical facts from the book of Ransford [15]. Let $D$ be a proper subdomain of $\mathbb{C} \cup\{\infty\}$ such that the boundary $\partial D$ (with respect to $\mathbb{C} \cup\{\infty\}$ ) is nonpolar. Let $\mathcal{P}_{D}$ be the Poisson projection of $D$, and $A$ a Borel subset of $\partial D$. Consider the bounded function

$$
1_{\partial D \backslash A}(\zeta):= \begin{cases}1, & \zeta \in \partial D \backslash A, \\ 0, & \zeta \in A .\end{cases}
$$

By Theorem 4.3.3 of [15], the harmonic measure of the set $\partial D \backslash A$ (or equivalently $h_{A, D}$ ) is exactly the Perron solution of the generalized Dirichlet problem with boundary data $1_{\partial D \backslash A}$. In other words,

$$
h_{A, D}=\mathcal{P}_{D}\left[1_{\partial D \backslash A}\right] \text {. }
$$

2.2. A uniqueness theorem and the Two-Constant Theorem. The following uniqueness theorem is very useful.

Theorem 2.1 (see [5]). Let $D \subset \mathbb{C}^{n}$ be a domain such that $D$ is locally $\mathcal{C}^{1}$ smooth on some open set $U$ of $\partial D$. If a set $E \subset D \cup U$ has a positive $(2 n-1)$-dimensional Hausdorff measure, then, for $f \in \mathcal{C}(D \cup U) \cap \mathcal{O}(D)$, $f=0$ on $E$ implies $f \equiv 0$.

Proof. The only nontrivial case is that $E \subset U$. In this case by taking the intersection of $D$ with a bundle of complex lines and applying the classical one-dimensional boundary uniqueness theorem of Privalov, one may find a set $E^{\prime} \subset D$ close to $E$ such that $E^{\prime}$ has a positive $2 n$-dimensional Hausdorff measure and $f=0$ on $E^{\prime}$. This completes the proof.

The following Two-Constant Theorem for plurisubharmonic functions will play a vital role in this paper.

THEOREM 2.2. If $u$ is a plurisubharmonic function in a bounded open set $D \subset \mathbb{C}^{n}$ and $u \leq M$ on $D$ and $\widehat{u} \leq m$ on some subset $A$ of $\bar{D}$, then

$$
\widehat{u}(z) \leq m(1-\omega(z, A, D))+M \omega(z, A, D), \quad z \in \bar{D} .
$$

Proof. This follows immediately from the definition of $\omega(\cdot, A, D)$ given in Subsection 1.1.

2.3. Uniform estimate for the Poisson kernels of a family of $\mathcal{C}^{2}$ smooth domains. In what follows we fix an integer $N \geq 2$ and let dist $(\cdot, \cdot)$ denote the Euclidean distance and $B(a, r)\left(a \in \mathbb{R}^{N}, r>0\right)$ the Euclidean ball of center $a$ and radius $r$. We say that a domain $D \subset \mathbb{R}^{N}$ is $\mathcal{C}^{2}$ smooth if $D$ is bounded and admits a defining function $\varrho \in \mathcal{C}^{2}\left(\mathbb{R}^{N}\right)$ such that $d \varrho(z) \neq 0$ for all $z \in \partial D$. Let $P_{D}$ denote its Poisson kernel. The following result is due to N. Kerzman (see [8] and [11]). 
TheOREM 2.3. Let $D \subset \mathbb{R}^{N}$ be a $\mathcal{C}^{2}$ smooth domain that satisfies

$$
\operatorname{diam}(D):=\sup _{x, y \in D}|x-y| \leq M \quad \text { for some constant } M .
$$

Then

(1) there is a positive number $r=r(D)$ such that for each $y \in \partial D$ there are balls $B\left(c_{y}, r\right) \subset D$ and $B\left(\widetilde{c}_{y}, r\right) \subset \mathbb{R}^{N} \backslash \bar{D}$ that satisfy

$$
\overline{B\left(\widetilde{c}_{y}, r\right)} \cap \bar{D}=\{y\}, \quad \overline{B\left(c_{y}, r\right)} \cap\left(\mathbb{R}^{N} \backslash D\right)=\{y\}
$$

(2) there is a constant $C$ which depends only on $N, r$, and $M$ such that

$$
P_{D}(x, y) \leq C \frac{\operatorname{dist}(x, \partial D)}{|x-y|^{N}}, \quad x \in D, y \in \partial D .
$$

Proof. This theorem is implicitly proved in Lemmas 8.2.3-8.2.5 and Proposition 8.2.6 of Krantz [11]. We only mention here that Kerzman's idea is to compare the Green function and the Poisson kernel for $D$ with the corresponding functions for the internally and externally tangent balls $B\left(c_{y}, r\right)$ and $B\left(\widetilde{c}_{y}, r\right)$ (and also for their complement).

Now we reformulate Kerzman's theorem in order to obtain a uniform upper bound for the Poisson kernels of a family of domains which depend, in some sense, continuously on a parameter.

Corollary 2.4. Let $\left(D_{\alpha}\right)_{\alpha \in I}$ be a family of $\mathcal{C}^{2}$ smooth domains in $\mathbb{R}^{N}$ indexed by a set I. Suppose that

(1) there is a constant $M$ such that for all $\alpha \in I$,

$$
\operatorname{diam}\left(D_{\alpha}\right) \leq M
$$

(2) there is a positive number $r$ such that for each $\alpha \in I, y \in \partial D_{\alpha}$, there are balls $B\left(c_{y, \alpha}, r\right) \subset D_{\alpha}$ and $B\left(\widetilde{c}_{y, \alpha}, r\right) \subset \mathbb{R}^{N} \backslash \bar{D}_{\alpha}$ that satisfy

$$
\overline{B\left(\widetilde{c}_{y, \alpha}, r\right)} \cap \bar{D}_{\alpha}=\{y\}, \quad \overline{B\left(c_{y, \alpha}, r\right)} \cap\left(\mathbb{R}^{N} \backslash D_{\alpha}\right)=\{y\} .
$$

Then there exists a constant $C$ such that

$$
P_{D_{\alpha}}(x, y) \leq C \frac{\operatorname{dist}\left(x, \partial D_{\alpha}\right)}{|x-y|^{N}}, \quad x \in D_{\alpha}, y \in \partial D_{\alpha}, \alpha \in I .
$$

Proof. This follows immediately from Theorem 2.3.

We conclude this section with an example of a family of $\mathcal{C}^{2}$ smooth domains satisfying the hypothesis of Corollary 2.4 .

Let $D$ be a domain in $\mathbb{C}^{n}$ which is locally $\mathcal{C}^{2}$ smooth on an open neighborhood of a point $P \in \partial D$. Let $T_{P}^{\mathbb{C}}\left(\right.$ resp. $\left.T_{P}^{\mathbb{R}}\right)$ denote the complex (resp. real) tangent hyperplane to $\partial D$ at $P$, and $\pi$ (resp. $\pi^{\mathbb{C}}$ ) the orthogonal projection from $\mathbb{C}^{n}$ onto $T_{P}^{\mathbb{R}}$ (resp. $T_{P}^{\mathbb{C}}$ ). By an affine transformation, we may suppose without loss of generality that $P=0, T_{P}^{\mathbb{C}}=\left\{z_{1}=0\right\}$ and $T_{P}^{\mathbb{R}}=\left\{\operatorname{Re} z_{1}=0\right\}$. 
Moreover, there are an open neighborhood $U$ of the origin and a function $\varrho \in \mathcal{C}^{2}(U)$ such that

$$
\varrho(0)=0, \quad d \varrho(0)=(1,0, \ldots, 0), \quad U \cap D=\{\varrho<0\} .
$$

For any domain $V \subset U$ and any $Q:=\left(0, z^{\prime}\right)=\left(0, z_{2}, \ldots, z_{n}\right) \in T_{P}^{\mathbb{C}}$, consider the planar domain

$$
V_{Q}:=\operatorname{env}\left(V \cap\left\{\left(t, z^{\prime}\right): t \in \mathbb{C}\right\}\right),
$$

where $\operatorname{env}(G)$ denotes the smallest simply connected domain containing (a given planar domain) $G$, in other words, $\operatorname{env}(G)$ is obtained from $G$ by adding all its holes.

Proposition 2.5. Under the above hypotheses and notation, there are open neighborhoods $U_{1}$ of $P$ in $T_{P}^{\mathbb{C}}, U_{2}$ of $P$ in $T_{P}^{\mathbb{R}}$ and $U_{3}$ of $P$ in $\mathbb{C}^{n}$ and $a \mathcal{C}^{2}$ smooth subdomain $V \subset D$ such that

(1) $U_{1}=U_{2} \cap T_{P}^{\mathbb{C}}$

(2) $\partial V \cap \partial D$ is an open neighborhood of $P$ in $\partial D$ and in $\partial V$, and $\pi$ is one-to-one from $\partial V \cap \partial D$ onto an open neighborhood of $U_{2}$;

(3) $\left(V_{Q}\right)_{Q \in U_{1}}$ is a family of $\mathcal{C}^{2}$ smooth planar simply connected domains which satisfies (1) and (2) of Corollary 2.4 and $V_{Q} \subset D$;

(4) there is a constant $C$ such that for all $Q \in U_{1}, z \in V_{Q} \cap U_{3}$ and $\zeta \in \partial V \cap \partial D$ satisfying $\pi(\zeta)=\pi(z)$,

$$
\operatorname{dist}\left(z, \partial V_{Q}\right) \leq C \operatorname{dist}(z, \partial D), \quad \operatorname{dist}(z, \zeta) \leq C \operatorname{dist}(z, \partial D) ;
$$

in other words, the quantities $\operatorname{dist}\left(z, \partial V_{Q}\right), \operatorname{dist}(z, \zeta)$ and $\operatorname{dist}(z, \partial D)$ are equivalent.

Proof. Since $D$ is locally $\mathcal{C}^{2}$ smooth on an open neighborhood of $P \in$ $\partial D$, a geometric argument (see [11, p. 325]) shows that there is an $r>0$ such that the sphere $\partial B$ is internally tangent to $D$ at $P$, where $B:=$ $B((-r, 0, \ldots, 0), r)$.

Consider the following defining function for $B$ :

$$
\phi(z):=\frac{\left(x_{1}+r\right)^{2}+y_{1}^{2}+\left|z^{\prime}\right|^{2}-r^{2}}{2 r}, \quad z=\left(x_{1}+i y_{1}, z^{\prime}\right) \in \mathbb{C}^{n} .
$$

A straightforward computation shows that $|d \phi|=1$ on $\partial B$. Next fix a radial function $\psi \in \mathcal{C}_{0}\left(\mathbb{C}^{n}\right)$ such that $0 \leq \psi \leq 1, \psi(z)=1$ for $|z| \leq 1$ and $\psi(z)=0$ for $|z| \geq 2$. Since $d \varrho(0)=d \phi(0)$, we may choose $\varepsilon_{0}$ such that $0<\varepsilon_{0}<r / 4$ and

$$
|(d \varrho-d \phi)(z)|<1 / 8, \quad|z| \leq 2 \varepsilon_{0} .
$$

Now define for any $0<\varepsilon<\varepsilon_{0}$,

$$
\psi_{\varepsilon}(z):=\psi(z / \varepsilon), \quad \varrho_{\varepsilon}:=\phi+\psi_{\varepsilon}(\varrho-\phi) .
$$


Observe that $\varrho_{\varepsilon}(z)=\varrho(z)$ for $|z| \leq \varepsilon$ and $\varrho_{\varepsilon}(z)=\phi(z)$ for $|z| \geq 2 \varepsilon$. Moreover using (2.5)-(2.6) and the identities $\varrho(0)=\phi(0)$ and $d \varrho(0)=d \phi(0)$, we have for $|z| \leq 2 \varepsilon$,

$$
\begin{aligned}
\left|\left(d \varrho_{\varepsilon}-d \phi\right)(z)\right| & \leq \psi_{\varepsilon}(z)|(d \varrho-d \phi)(z)|+\left|d \psi_{\varepsilon}(z)\right| \cdot|(\varrho-\phi)(z)| \\
& \leq \frac{1}{8}+\frac{C^{\prime} \varepsilon^{2}}{\varepsilon},
\end{aligned}
$$

where $C^{\prime}$ is a constant. Therefore there exists $\varepsilon_{1}>0$ such that for all $0<\varepsilon<\varepsilon_{1}$,

$$
\left|\left(d \varrho_{\varepsilon}-d \phi\right)(z)\right| \leq 1 / 4, \quad|z| \leq 2 \varepsilon .
$$

For any $0<\varepsilon<\min \left\{\varepsilon_{0}, \varepsilon_{1}\right\}$ define

$$
V^{\prime}:=\left\{z \in \mathbb{C}^{n}: \varrho_{\varepsilon}(z)<0\right\}
$$

and let $V$ be the connected component of $V^{\prime}$ satisfying $P \in \partial V$. Then (2.7) implies that $\left|d \varrho_{\varepsilon}(z)\right|>1 / 2$ for $|z| \leq 2 \varepsilon$. Since $0 \leq \psi \leq 1$ and $\varrho_{\varepsilon}(z)=\phi(z)$ for $|z| \geq 2 \varepsilon$, we deduce from (2.6) that $V$ is a $\mathcal{C}^{2}$ smooth subdomain of $D$.

Now let

$$
U_{3}:=B(0, \varepsilon), \quad U_{2}:=U_{3} \cap T_{P}^{\mathbb{R}}, \quad U_{1}:=U_{3} \cap T_{P}^{\mathbb{C}} .
$$

Then by (2.4)-(2.6), we see that (1) and (2) are satisfied when $\varepsilon$ in (2.8) is sufficiently small.

We next turn to (3). Fix $Q \in U_{1}$ and $z \in \partial V_{Q}$. Then there are two cases. If $|z| \leq 2 \varepsilon$, then by $(2.7)$,

$$
\left|d_{z_{1}} \varrho_{\varepsilon}\right| \geq\left|d_{z_{1}} \phi\right|-\left|d \varrho_{\varepsilon}-d \phi\right|>\frac{\left|z_{1}+r\right|}{r}-\frac{1}{4}>\frac{1}{4} .
$$

If $|z| \geq 2 \varepsilon$, then by $(2.6)$,

$$
\left|d_{z_{1}} \varrho_{\varepsilon}\right|=\left|d_{z_{1}} \phi\right|=\frac{\left|z_{1}+r\right|}{r}>0 .
$$

Thus for any $Q=\left(0, z^{\prime}\right) \in U_{1}$ the set $V \cap\left\{\left(t, z^{\prime}\right): t \in \mathbb{C}\right\}$ is a $\mathcal{C}^{2}$ smooth planar region contained in $D$. Since for sufficiently small $\varepsilon>0, \partial D \cap U_{3}$ is a graph over $T_{P}^{\mathbb{R}}$, a geometric argument shows that $V_{Q}$ is also a $\mathcal{C}^{2}$ smooth planar simply connected region contained in $D$. We see that one may assume that $V_{Q}$ is a domain.

To complete the proof of (3) we still need to check that the family $\left(V_{Q}\right)_{Q \in U_{1}}$ satisfies (1) and (2) of Corollary 2.4. Indeed, let $\varrho_{Q}$ be the restriction of $\varrho$ to the complex line containing $V_{Q}$. Clearly, the Hessian $d^{2} \varrho_{Q}$ depends continuously on $Q \in U_{1}$. This, combined with the geometric fact proved in [11, p. 325], implies the remaining assertion of (3).

It remains to establish (4). Also by [11, p. 325], when $\varepsilon>0$ in (2.8) is sufficiently small, for any $z \in U_{3} \cap D$ there are unique $\theta \in \partial D$ and a point 
$\eta \in \partial V_{Q}$ such that

$$
|z-\theta|=\operatorname{dist}(z, \partial D), \quad|z-\eta|=\operatorname{dist}\left(z, \partial V_{Q}\right) .
$$

Let $n_{\theta}$ (resp. $\left.n_{\eta}\right)$ be the inward unit normal vector to $\partial D\left(\operatorname{resp} . \partial V_{Q}\right)$ at $\theta$ (resp. $\eta$ ). Then a geometric argument shows that the orthogonal projection of the real line containing $n_{\eta}$ onto $V_{Q}$ passes through $z$. Since $Q$ is close to $P$, the angles between the vectors $z-\eta$ and $n_{\eta}$ and between $n_{\eta}$ and $n_{\theta}$ are arbitrarily small when $\varepsilon$ in (2.8) is sufficiently small. Thus the angle between $z-\eta$ and $z-\theta$ is arbitrarily small. Since $|d \varrho(0)|=1$ and $\varrho \in \mathcal{C}^{2}(U)$, it follows that

$$
|z-\theta| \leq|z-\eta| \leq C|z-\theta|
$$

for some constant $C$, which proves that $\operatorname{dist}\left(z, \partial V_{Q}\right) \leq C \operatorname{dist}(z, \partial D)$.

The second estimate of (4) can be proved in exactly the same way. This completes the proof.

3. Estimates for the plurisubharmonic measures. In this section we apply the result of the previous one to establish some inequalities concerning plurisubharmonic measures. These estimates will be crucial for the proof of the Main Theorem.

Proposition 3.1. Let $D$ be a bounded planar domain with $\mathcal{C}^{2}$ smooth boundary. Then there is a constant $C$ such that for any finite union $A$ of open connected arcs on $\partial D$,

$$
\omega(z, A, D) \leq C \frac{\operatorname{dist}(z, \partial D)}{\operatorname{dist}(z, \partial D \backslash A)^{2}}, \quad z \in D \cup A .
$$

Proof. By Theorem 2.3 we know that there is a constant $C^{\prime}$ such that

$$
P_{D}(x, y) \leq C^{\prime} \frac{\operatorname{dist}(x, \partial D)}{|x-y|^{2}}, \quad x \in D, y \in \partial D
$$

This, combined with identity (2.1), implies that

$$
\omega(z, A, D) \leq C^{\prime} \int_{\partial D \backslash A} \frac{\operatorname{dist}(z, \partial D)}{|z-\zeta|^{2}} d \sigma(\zeta),
$$

where $d \sigma$ is the Lebesgue measure on $\partial D$. We easily see that the right side of the latter estimate is dominated by

$$
C \frac{\operatorname{dist}(z, \partial D)}{\operatorname{dist}(z, \partial D \backslash A)^{2}}
$$

Observe that as in Theorem 2.3 and Corollary 2.4, the constant $C$ in Proposition 3.1 depends only on $\sigma(\partial D \backslash A)$, diam $(D)$ and the radius $r(D)$.

Proposition 3.2. Let $D \subset \mathbb{C}^{n}$ be a bounded open set and let $A$ be an open subset of $\partial D$ such that $D$ is locally $\mathcal{C}^{2}$ smooth on $A$. Then for any set 
$K \Subset A$, there is a constant $C=C_{K}$ such that

$$
\omega(z, A, D) \leq C \operatorname{dist}(z, K), \quad z \in D \cup A .
$$

In particular, $\omega(\cdot, A, D)=0$ on $A$.

Proof. Since $\omega(\cdot, A, D) \leq \omega(\cdot, B, G)$ if $B \subset A$ and $G \subset D$, by a compactness argument and Proposition 2.5 we may suppose that $K \Subset A$ is the intersection of $A$ and a sufficiently small ball $U$ centered at $P$, and $D$ is a $\mathcal{C}^{2}$ smooth domain such that Proposition 2.5 is applicable. Namely, keeping the notation of (2.2) and (2.3), we assume that $P=0 \in \mathbb{C}^{n}$ and $\left(V_{Q}\right)_{Q \in U_{1}}$ is a family of $\mathcal{C}^{2}$ smooth planar simply connected domains satisfying (1) and (2) of Corollary 2.4.

Observe that it suffices to prove the proposition for the case where $z$ is sufficiently close to $K$. Now let $Q:=\pi^{\mathbb{C}}(z)$ and note that $z \in D_{Q}$. Then Proposition 2.5(4) gives a constant $C^{\prime}$ such that

$$
\operatorname{dist}(z, K) \leq \operatorname{dist}\left(z, \partial V_{Q}\right) \leq C^{\prime} \operatorname{dist}(z, K) .
$$

Combining Propositions 2.5, 3.1 and the remark following the proof of Proposition 3.1, we see that there is a constant $C^{\prime \prime}$ such that

$$
\omega\left(z, A \cap \partial V_{Q}, V_{Q}\right) \leq C^{\prime \prime} \operatorname{dist}\left(z, K \cap \partial V_{Q}\right) .
$$

Next observe that for any $u \in \mathcal{P S H}(D)$ with $u \leq 1$ on $D$ and $\widehat{u} \leq 0$ on $A$, by the very definition,

$$
u(z) \leq \omega\left(z, A \cap \partial V_{Q}, V_{Q}\right) .
$$

Combined with (3.1) and (3.2), this implies that

$$
\omega(z, A, D) \leq C^{\prime} C^{\prime \prime} \operatorname{dist}(z, K),
$$

which completes the proof of the desired estimate. The identity $\omega(\cdot, A, D)$ $=0$ on $A$ follows immediately from this estimate.

The next result tells us that the definition of the plurisubharmonic measure given in Case II of Subsection 1.1 is independent of the choices made.

Proposition 3.3. Let $D \subset \mathbb{C}^{n}$ be an open set and let $A$ be an open subset of $\partial D$ such that $D$ is locally $\mathcal{C}^{2}$ smooth on $A$. Then there is a function $\omega(\cdot, A, D)$ plurisubharmonic in $D$ with the following property:

Let $\left(D_{k}\right)_{k=1}^{\infty}$ be a sequence of relatively compact open sets $D_{k} \Subset \mathbb{C}^{n}$ and $\left(A_{k}\right)_{k=1}^{\infty}$ a sequence of open subsets of $A$ such that

(i) $D_{k} \subset D_{k+1}$ and $\bigcup_{k=1}^{\infty} D_{k}=D$;

(ii) $A_{k} \subset A_{k+1}$ and $A_{k} \subset \partial D \cap \partial D_{k}$ and $\bigcup_{k=1}^{\infty} A_{k}=A$;

(iii) for any $\zeta \in A$ there is an open neighborhood $V=V_{\zeta}$ of $\zeta$ in $\mathbb{C}^{n}$ such that $V \cap D=V \cap D_{k}$ for some $k$.

Then

$$
\omega(\cdot, A, D)=\lim _{k \rightarrow \infty} \omega\left(\cdot, A_{k}, D_{k}\right) \quad \text { on } D .
$$


Proof. First observe that such sequences $\left(D_{k}\right)_{k=1}^{\infty}$ and $\left(A_{k}\right)_{k=1}^{\infty}$ always exist. For example, one may take $D_{k}:=D \cap B(0, k)$ and $A_{k}:=A \cap B(0, k)$, $k \in \mathbb{N}$. Let $\left(D_{k}^{\prime}\right)_{k=1}^{\infty}$ and $\left(A_{k}^{\prime}\right)_{k=1}^{\infty}$ be some other sequences which satisfy (i)-(iii). It is easy to see that the limits of decreasing sequences

$$
u:=\lim _{k \rightarrow \infty} \omega\left(\cdot, A_{k}, D_{k}\right) \quad \text { and } \quad u^{\prime}:=\lim _{k \rightarrow \infty} \omega\left(\cdot, A_{k}^{\prime}, D_{k}^{\prime}\right)
$$

exist and define two plurisubharmonic functions in $D$.

Fix $k$ and let $\zeta$ be any point in $A_{k}$ and $K$ be any compact neighborhood of $\zeta$ in $A_{k}$. By (i)-(iii), there are an integer $N$ and a bounded open neighborhood $U$ of $K$ in $\mathbb{C}^{n}$ such that $U \cap A \subset A_{n}^{\prime}$ and $U \cap D_{n}^{\prime}=U \cap D$ for any $n \geq N$. Therefore, applying Proposition 3.2 to the open set $D \cap U$, we find a constant $C$ such that

$$
\omega\left(z, A_{n}^{\prime}, D_{n}^{\prime}\right) \leq \omega(z, U \cap A, U \cap D) \leq C \operatorname{dist}(z, K), \quad z \in U \cap D, n \geq N .
$$

This implies that

$$
\widehat{u^{\prime}}(\zeta):=\limsup _{w \in D, w \rightarrow \zeta} u^{\prime}(w)=0 .
$$

Thus $\widehat{u^{\prime}}=0$ on $A_{k}$ and therefore $\omega\left(\cdot, A_{k}, D_{k}\right) \geq u^{\prime}$. This implies that $u \geq u^{\prime}$. Similarly, one gets $u^{\prime} \geq u$. Hence $u=u^{\prime}$ and the proof is finished.

One should mention that in view of Proposition 3.3, Proposition 3.2 still holds when $D$ is an arbitrary (not necessarily bounded) open set. An immediate consequence of Proposition 3.3 is the following result.

Proposition 3.4. Let $D \subset \mathbb{C}^{n}$ be an open set and let $A$ be an open subset of $\partial D$ such that $D$ is locally $\mathcal{C}^{2}$ smooth on $A$. Let $\left(A_{k}\right)_{k=1}^{\infty}$ be a sequence of open subsets of $\partial D$ such that $A_{k} \nearrow A$ as $k \nearrow \infty$. Then

$$
\lim _{k \rightarrow \infty} \omega\left(\cdot, A_{k}, D\right)=\omega(\cdot, A, D) \quad \text { on } D .
$$

Proof. It suffices to take $\left(D_{k}\right)_{k=1}^{\infty}$ with $D_{k}=D$, and apply Proposition 3.3.

Proposition 3.5. Let $D \subset \mathbb{C}^{n}$ be an open set and let $A$ be an open subset of $\partial D$ such that $D$ is locally $\mathcal{C}^{2}$ smooth on $A$. Then for any $\delta>0$, there is an open subset $T_{\delta}$ of $D$ such that

(1) $T_{\delta_{1}} \subset T_{\delta_{2}}$ for $0<\delta_{1}<\delta_{2}$;

(2) $T_{\delta} \cup A$ is an open neighborhood of $A$ in $A \cup D$;

(3) $\omega(z, A, D)-\delta \leq \omega\left(z, T_{\delta}, D\right) \leq \omega(z, A, D)$ for $z \in D$;

(4) $\sup _{T_{\delta}} \operatorname{dist}(\cdot, A)<\delta$.

Proof. Fix a sequence $\left(A_{k}\right)_{k=1}^{\infty}$ of open subsets of $A$ such that

(i) $A_{k} \Subset A_{k+1} \subset A$;

(ii) $A_{k} \nearrow A$ as $k \nearrow \infty$;

(iii) $A_{k}$ consists of a finite number of open connected components. 
By Propositions 3.2 and 3.3, for any $k$ there is a constant $C_{k}>1$ such that

$$
\omega(z, A, D) \leq C_{k} \operatorname{dist}\left(z, A_{k}\right) .
$$

For $\delta>0$ consider the following open subset of $D$ :

$$
T_{\delta}:=\left\{z \in D: C_{k} \operatorname{dist}\left(z, A_{k}\right)<\delta \text { for some } k \in \mathbb{N}\right\}
$$

In view of (3.3)-(3.4) and (i)-(iii), statements (1) and (2) are proved. Moreover

$$
\omega(z, A, D) \leq \delta, \quad \operatorname{dist}(z, A)<\delta, \quad z \in T_{\delta},
$$

which implies that $\omega(\cdot, A, D)-\delta \leq \omega\left(\cdot, T_{\delta}, D\right)$ on $D$.

On the other hand, from (2) and the definition of plurisubharmonic measures, we deduce that $\omega\left(\cdot, T_{\delta}, D\right) \leq \omega(\cdot, A, D)$ on $D$. Hence the proof is complete.

The rest of this section is devoted to some applications of the previous results.

Proposition 3.6. Let $D \subset \mathbb{C}^{n}$ and $G \subset \mathbb{C}^{m}$ be two domains and let $A($ resp. B) be an open subset of $\partial D($ resp. $\partial G)$ such that $D$ (resp. $G)$ is locally $\mathcal{C}^{2}$ smooth on $A$ (resp. B). Put $X:=\mathbb{X}(A, B ; D, G)$ and $\widehat{X}^{\circ}:=$ $\widehat{\mathbb{X}}^{\circ}(A, B ; D, G)$. Then

(1) for any finite subset $M \subset \widehat{X}^{\circ}$, there are open sets $T \subset D, S \subset G$ and $0<\varepsilon<1$ such that

$$
M \subset\{(z, w) \in D \times G: \omega(z, T, D)+\omega(w, S, G)<1-\varepsilon\} \subset \widehat{X}^{\circ} ;
$$

(2) the open set $\widehat{X}^{\mathrm{o}}$ is connected;

(3) $X \subset \widehat{X}$.

Proof. Fix $\varepsilon>0$ such that

$$
\omega(z, A, D)+\omega(w, B, G)<1-2 \varepsilon, \quad(z, w) \in M .
$$

Applying Proposition 3.5, we find two open sets $T \subset D, S \subset G$ of the form (3.4) such that

$$
\begin{aligned}
|\omega(z, A, D)-\omega(z, T, D)|<\varepsilon / 2, & z \in D \\
|\omega(w, B, G)-\omega(w, S, G)|<\varepsilon / 2, & w \in G .
\end{aligned}
$$

Therefore,

$$
M \subset\{(z, w) \in D \times G: \omega(z, T, D)+\omega(w, S, G)<1-\varepsilon\} \subset \widehat{X}^{\mathrm{o}},
$$

which yields (1).

To prove $(2)$ let $\left(z_{1}, w_{1}\right)$ and $\left(z_{2}, w_{2}\right)$ be two arbitrary points in $\widehat{X}^{\mathrm{o}}$. Put $M:=\left\{\left(z_{1}, w_{1}\right),\left(z_{2}, w_{2}\right)\right\}$. By (1) there are open sets $T \subset D, S \subset G$ and $0<\varepsilon<1$ such that

$$
M \subset\{(z, w) \in D \times G: \omega(z, T, D)+\omega(w, S, G)<1-\varepsilon\} \subset \widehat{X}^{\mathrm{o}} .
$$


Since the middle set above is connected (see, for example, Lemma 4 in [7]), the conclusion of (2) follows.

Statement (3) holds by Proposition 3.2 and the remark following the proof of Proposition 3.3.

The next result tells us that the open set $\widehat{X}^{\text {o }}$ is still connected in the following mixed situation.

Proposition 3.7. Let $D \subset \mathbb{C}^{n}$ and $G \subset \mathbb{C}^{m}$ be two domains, let $A \subset D$ and let $B$ be an open subset of $\partial G$. Assume that $A$ is locally pluriregular and $G$ is locally $\mathcal{C}^{2}$ smooth on $B$. Let $X:=\mathbb{X}(A, B ; D, G)$ and $\widehat{X}^{\circ}:=\widehat{\mathbb{X}}^{\circ}(A, B ; D, G)$. Then

(1) for any finite subset $M \subset \widehat{X}^{\circ}$, there are an open set $S \subset G$ and $0<\varepsilon<1$ such that

$$
M \subset\{(z, w) \in D \times G: \omega(z, A, D)+\omega(w, S, G)<1-\varepsilon\} \subset \widehat{X}^{\circ} ;
$$

(2) the open set $\widehat{X}^{\mathrm{o}}$ is connected;

(3) $X \subset \widehat{X}$.

Proof. Proceed as in the proof of Proposition 3.6 with obvious changes.

The last result of this section studies the level sets of plurisubharmonic measures.

Proposition 3.8. Let $D \subset \mathbb{C}^{n}$ be an open set and let $A$ be an open subset of $\partial D$ such that $D$ is locally $\mathcal{C}^{2}$ smooth on $A$. For any $0<\varepsilon<1$ let

$$
D_{\varepsilon}:=\{z \in D: \omega(z, A, D)<1-\varepsilon\} .
$$

Then

(1) we have

$\lim _{\varepsilon \rightarrow 0} \omega\left(\cdot, A, D_{\varepsilon}\right)=\omega(\cdot, A, D) \quad$ on $D, \quad \omega\left(\cdot, A, D_{\varepsilon}\right)=\frac{\omega(\cdot, A, D)}{1-\varepsilon} \quad$ on $D_{\varepsilon} ;$

(2) if $z \in D$ satisfies $\omega(z, A, D)<1$, then, for any $0<\varepsilon<1-$ $\omega(z, A, D)$, the connected component of $D_{\varepsilon}$ which contains $z$ is locally $\mathcal{C}^{2}$ smooth on a nonempty open subset of $A$;

(3) for any $0<\varepsilon_{0}<1$, there is an open neighborhood $U$ of $A$ in $D \cup A$ such that for all $\varepsilon \leq 1-\varepsilon_{0}$ there exists exactly one connected component of $D_{\varepsilon}$ containing $U \cap D$ and satisfying $\omega\left(\cdot, A, D_{\varepsilon}\right)<\varepsilon_{0}$ on $U$.

Proof. For $k \in \mathbb{N}$ let $A_{k}:=A$. It suffices to check that the sequences $\left(D_{1 / k}\right)_{k=N}^{\infty}$ and $\left(A_{k}\right)_{k=N}^{\infty}$ have properties (i)-(iii) of Proposition 3.3 for a sufficiently large positive integer $N$. Observe that the only nontrivial point is (iii), which however follows immediately from Proposition 3.2. Hence the first identity of (1) is proved. To verify the second, observe that $\omega\left(\cdot, A, D_{\varepsilon}\right) \geq$ 
$\omega(\cdot, A, D) /(1-\varepsilon)$ on $D_{\varepsilon}$ by the very definition. On the other hand, consider the function

$$
u(z):= \begin{cases}\max \left\{\omega(z, A, D),(1-\varepsilon) \omega\left(z, A, D_{\varepsilon}\right)\right\}, & z \in D_{\varepsilon}, \\ \omega(z, A, D), & z \in D \backslash D_{\varepsilon} .\end{cases}
$$

Clearly, $u \in \mathcal{P S H}(D)$ and $u \leq 1$ on $D$. By Proposition 3.2, $\widehat{u}=0$ on $A$. Thus $u \leq \omega(\cdot, A, D)$, which completes the proof of the second identity of (1).

By (1), $\omega\left(z, A, D_{\varepsilon}\right)<1$ for all $0<\varepsilon<1-\omega(z, A, D)$. This proves (2).

For (3) it suffices to show that every $\zeta \in A$ has a neighborhood $U$ in $D \cup A$ with the required properties. By Proposition 3.2 one may find an open neighborhood $\mathcal{U}$ of $\zeta$ such that for some constant $C_{1}$,

$$
\omega(\cdot, A, D) \leq C_{1} \operatorname{dist}(z, \mathcal{U} \cap A)<\varepsilon_{0} \quad \text { on } \mathcal{U} \cap D .
$$

This shows that $\mathcal{U} \cap D \subset D_{1-\varepsilon_{0}}$. We now choose a relatively compact neighborhood $U$ of $\zeta$ such that $U \Subset \mathcal{U}$. Then applying Proposition 3.2 and shrinking $U$ if necessary, we also have

$$
\omega\left(\cdot, A, D_{1-\varepsilon_{0}}\right) \leq C_{2} \operatorname{dist}(z, A)<\varepsilon_{0} \quad \text { on } U \cap D,
$$

which completes the proof of the last assertion.

\section{A mixed cross theorem and two quantitative cross theorems.} The main result of this section is the following mixed cross theorem.

Theorem 4.1. Let $D \subset \mathbb{C}^{n}$ be a bounded pseudoconvex domain, $G \subset \mathbb{C}^{m}$ a domain, $A \subset D$, and $B \subset \partial G$. Assume that $A$ is a locally pluriregular relatively compact subset of $D$ and $A=\bigcup_{k=1}^{\infty} A_{k}$ with $A_{k}$ locally pluriregular and compact; assume moreover that $B$ is an open subset of $\partial G$ such that $G$ is locally $\mathcal{C}^{2}$ smooth on $B$. Let $X:=\mathbb{X}(A, B ; D, G), X^{\circ}:=\mathbb{X}^{\circ}(A, B ; D, G)$, $\widehat{X}:=\widehat{\mathbb{X}}(A, B ; D, G)$, and $\widehat{X}^{\mathrm{o}}:=\widehat{\mathbb{X}}^{\circ}(A, B ; D, G)$.

Let $\mathcal{C}_{\mathrm{s}}(X)$ be the space of all functions defined on $X$ such that

(i) $f$ is locally bounded on $X$;

(ii) for any $z \in A, f(z, \cdot) \in \mathcal{C}(G \cup B)$. Then

(1) for any $f \in \mathcal{C}_{\mathrm{s}}(X) \cap \mathcal{O}_{\mathrm{s}}\left(X^{\mathrm{o}}\right)$ there is a unique $\widehat{f} \in \mathcal{C}(\widehat{X}) \cap \mathcal{O}\left(\widehat{X}^{\mathrm{o}}\right)$ such that $\widehat{f}=f$ on $X$;

(2) if, moreover, there is a set $B^{\prime} \subset \partial G$ such that

(i') $f$ is locally bounded on $A \times\left(G \cup B^{\prime}\right)$,

(ii') for any $z \in A, f(z, \cdot) \in \mathcal{C}\left(G \cup B^{\prime}\right)$,

(iii') $\omega(\cdot, B, G) \in \mathcal{C}\left(G \cup B^{\prime}\right)$,

then $\widehat{f}$ extends continuously to every point $(z, \eta) \in \overline{\widehat{X}} \cap\left(D \times B^{\prime}\right)$.

A remark is in order. Under the hypothesis of Theorem 4.1, it follows from Proposition 3.7(3) that $X \subset \widehat{X}$. 
Proof. First we prove (1). We argue as in the proof of Theorem 3.5.1 in [6]. For completeness, we give a sketch. Fix an $f \in \mathcal{C}_{\mathrm{s}}(X) \cap \mathcal{O}_{\mathrm{s}}\left(X^{\mathrm{o}}\right)$.

SteP I: Reduction to the case where $D$ is strongly pseudoconvex, $A$ is a locally pluriregular compact subset of $D$ and $|f|$ is bounded on $X$.

One proceeds as in the first and second step of the proof in [6]. More precisely, let $\left(G_{k}\right)_{k=1}^{\infty}$ be an exhausting sequence for $G$ with properties (i)(iii) of Proposition 3.3 (with $B$ instead of $A$ ). Let $B_{k}:=B \cap \partial G_{k}$. Since $D$ is a domain of holomorphy, we may find an exhausting sequence $\left(D_{k}\right)_{k=1}^{\infty}$ of relatively compact, strongly pseudoconvex subdomains of $D$ with $A_{k} \subset$ $D_{k} \nearrow D$.

By reduction assumption, for each $k$ there exists an $\widehat{f}_{k} \in \mathcal{C}\left(\widehat{\mathbb{X}}\left(A_{k}, B_{k}\right.\right.$; $\left.\left.D_{k}, G_{k}\right)\right) \cap \mathcal{O}\left(\widehat{\mathbb{X}}^{\circ}\left(A_{k}, B_{k} ; D_{k}, G_{k}\right)\right)$ such that $\widehat{f}_{k}=f$ on $\mathbb{X}\left(A_{k}, B_{k} ; D_{k}, G_{k}\right)$.

By Theorem 2.1 and Proposition 3.7 and since $f_{k+1}=f_{k}=f$ on $\mathbb{X}\left(A_{k}, B_{k} ; D_{k}, G_{k}\right)$, one can show that $f_{k+1}=f_{k}=f$ on $\widehat{\mathbb{X}}\left(A_{k}, B_{k} ; D_{k}, G_{k}\right)$. On the other hand, by Proposition $3.3, \widehat{\mathbb{X}}\left(A_{k}, B_{k} ; D_{k}, G_{k}\right) \nearrow \widehat{\mathbb{X}}$ as $k \nearrow \infty$. Therefore, we may glue $f_{k}$ together to obtain $\widehat{f} \in \mathcal{C}(\widehat{X}) \cap \mathcal{O}\left(\widehat{X}^{\circ}\right)$ such that $\widehat{f}=\widehat{f_{k}}=f$ on $\mathbb{X}\left(A_{k}, B_{k} ; D_{k}, G_{k}\right)$. Thus $\widehat{f}=f$ on $X$. The uniqueness of $\widehat{f}$ follows from Theorem 2.1 and Proposition 3.7. This completes Step I.

STEP II: The case where $D$ is strongly pseudoconvex, $A$ is a locally pluriregular compact subset of $D$ and $|f| \leq 1$ on $X$.

The key observation is that we are still able to apply the classical method of doubly orthogonal bases of Bergman type (see for example [12], [13] for a systematic study of this method).

Next one observes that Lemma 3.5.10 of [6] is still valid in the present context. Look at Step 3 in that proof. In what follows, we will use the notation from [6].

Let $\mu:=\mu_{A, D}, H_{0}:=L_{h}^{2}(D), H_{1}:=$ the closure of $\left.H_{0}\right|_{A}$ in $L^{2}(A, \mu)$ and let $\left(b_{k}\right)_{k=1}^{\infty}$ be the basis from Lemma 3.5.10 in [6], $\nu_{k}:=\left\|b_{k}\right\|_{H_{0}}, k \in \mathbb{N}$, and $\nu_{k} \nearrow \infty$. For any $w \in B$, we have $f(\cdot, w) \in H_{0}$ and $\left.f(\cdot, w)\right|_{A} \in H_{1}$. Hence

$$
f(\cdot, w)=\sum_{k=1}^{\infty} c_{k}(w) b_{k}
$$

where

$$
c_{k}(w)=\frac{1}{\nu_{k}^{2}} \int_{D} f(z, w) \overline{b_{k}(z)} d \Lambda_{2 n}(z)=\int_{A} f(z, w) \overline{b_{k}(z)} d \mu(z), \quad k \in \mathbb{N} .
$$

Since $|f| \leq 1$ on $X$ and $f \in \mathcal{C}_{\mathrm{s}}(X) \cap \mathcal{O}_{\mathrm{s}}(X)$, applying Lebesgue's Dominated Convergence Theorem, we see that the formula 


$$
\widehat{c}_{k}(w):=\int_{A} f(z, w) \overline{b_{k}(z)} d \mu(z), \quad w \in G \cup B, k \in \mathbb{N},
$$

defines a bounded function which is holomorphic in $G$. Moreover, from (4.2)(4.3) it follows that

$$
\lim _{w \in G, w \rightarrow \eta} \widehat{c}_{k}(w)=\widehat{c}_{k}(\eta)=c_{k}(\eta), \quad \eta \in B .
$$

Thus $\widehat{c}_{k} \in \mathcal{C}(G \cup B) \cap \mathcal{O}(G)$.

Observe that as in [6], using (4.2)-(4.4), we obtain the following estimates:

$$
\begin{aligned}
& \frac{\log \left|\widehat{c}_{k}\right|}{\log \nu_{k}} \leq \frac{\sqrt{\mu(A)}}{\log \nu_{k}}, \quad k \in \mathbb{N}, \\
& \limsup _{w \in G, w \rightarrow \eta} \frac{\log \left|\widehat{c}_{k}(\eta)\right|}{\log \nu_{k}} \leq \frac{\log \sqrt{\Lambda_{2 n}(D)}}{\log \nu_{k}}-1, \quad \eta \in B, k \in \mathbb{N} .
\end{aligned}
$$

This shows that for any $\varepsilon>0$, there is an $N$ such that for all $k \geq N$,

$$
\frac{\log \left|\widehat{c}_{k}\right|}{\log \nu_{k}} \leq \omega(\cdot, B, G)+\varepsilon-1 \quad \text { on } G .
$$

Take a compact $K \Subset D$, let $\alpha>\max _{K} h_{A, D}^{*}$, and let $\varepsilon>0$ be so small that $\alpha+2 \varepsilon<1$. Consider the open set

$$
G_{K}:=\{w \in G: \omega(\cdot, B, G)<1-\alpha-2 \varepsilon\} .
$$

By (4.5) there is a constant $C^{\prime}(K)$ such that

$$
\left\|\widehat{c}_{k}\right\| \leq C^{\prime}(K) \nu_{k}^{\omega(\cdot, B, G)+\varepsilon-1} \leq C^{\prime}(K) \nu_{k}^{-\alpha-\varepsilon}, \quad k \geq 1 .
$$

Now we wish to show that

$$
\sum_{k=1}^{\infty} \widehat{c}_{k}(w) b_{k}(z)
$$

converges locally uniformly in $\widehat{X}^{\text {o }}$. Indeed, by (4.6) and Lemma 3.5.10 in [6],

$$
\begin{aligned}
\sum_{k=1}^{\infty}\left\|\widehat{c}_{k}\right\|_{G_{K}}\left\|b_{k}\right\|_{K} & \leq \sum_{k=1}^{\infty} C^{\prime}(K) \nu_{k}^{-\alpha-\varepsilon} C(K, \alpha) \nu_{k}^{\alpha} \\
& \leq C^{\prime}(K) C(K, \alpha) \sum_{k=1}^{\infty} \nu_{k}^{-\varepsilon}<\infty
\end{aligned}
$$

which gives the normal convergence on $K \times G_{K}$. Moreover, $B \subset \partial G_{K}$ by Proposition 3.2. Therefore, the previous argument also shows that the series in (4.7) converges normally on $K \times\left(G_{K} \cup B\right)$. Since the compact set $K \Subset D$ and $\varepsilon>0$ are arbitrary, the series in (4.7) converges uniformly on compact subsets of $\widehat{X}$. Let $\widehat{f}$ be its sum. Then obviously $\widehat{f} \in \mathcal{C}(\widehat{X}) \cap \mathcal{O}\left(\widehat{X}^{\circ}\right)$. From 
(4.1), (4.4) and (4.7) it follows that

$$
\widehat{f}=f \quad \text { on } D \times B .
$$

Consequently, an application of Theorem 2.1 shows that $\widehat{f}=f$ on $X$. This proves (1).

We now turn to (2) using the proof of (1). Observe that by (4.3) and $\left(\mathrm{i}^{\prime}\right)-\left(\mathrm{ii}^{\prime}\right), \widehat{c}_{k} \in \mathcal{C}\left(G \cup B^{\prime}\right) \cap \mathcal{O}(G)$. Next fix $\eta \in B^{\prime}$ and $z \in D$. We use hypothesis (iii') to choose $\varepsilon>0$ and a compact neighborhood $K$ of $z$ such that $K \times\left(G_{K} \cup\{\eta\}\right)$ is a neighborhood of $(z, \eta)$ in $\widehat{X} \cap\left(D \times B^{\prime}\right)$. The rest of the proof goes essentially along the same lines as that of (1).

The last two results of this section give quantitative versions of the classical cross theorem (cf. Theorem 1).

Theorem 4.2. Let $D \subset \mathbb{C}^{n}$ and $G \subset \mathbb{C}^{m}$ be bounded domains and let $A \subset D$ and $B \subset G$ be locally pluriregular sets. Assume that $D$ is pseudoconvex and $A$ is of the form $A=\bigcup_{k=1}^{\infty} A_{k}$ with $A_{k}$ compact. Let $X:=\mathbb{X}(A, B ; D, G)$ and $\widehat{X}:=\widehat{\mathbb{X}}(A, B ; D, G)$. Then for any $f \in \mathcal{O}_{\mathrm{s}}(X)$ there is a unique $\widehat{f} \in \mathcal{O}(\widehat{X})$ such that $\widehat{f}=f$ on $X$. Moreover, if $|f|_{X}<\infty$ then

$$
|\widehat{f}(z, w)| \leq|f|_{A \times B}^{1-\omega(z, A, D)-\omega(w, B, G)}|f|_{X}^{\omega(z, A, D)+\omega(w, B, G)}, \quad(z, w) \in \widehat{X} .
$$

Proof. We proceed in two steps.

SteP 1: Proof of the equality $|\widehat{f}|_{\widehat{X}}=|f|_{X}$.

Suppose that there is $\left(z_{0}, w_{0}\right) \in \widehat{X}$ such that $\left|\widehat{f}\left(z_{0}, w_{0}\right)\right|>|f|_{X}$. Put $\alpha:=\widehat{f}\left(z_{0}, w_{0}\right)$ and consider the function

$$
g(z, w):=\frac{1}{f(z, w)-\alpha}, \quad(z, w) \in X .
$$

Clearly, $g \in \mathcal{O}_{\mathrm{s}}(X)$. Hence by Theorem 3.5.1 of [6], there is exactly one $\widehat{g} \in \mathcal{O}(\widehat{X})$ with $\widehat{g}=g$ on $X$. Therefore, by (4.9) we have $g(f-\alpha) \equiv 1$ on $X$. Thus $\widehat{g}(\widehat{f}-\alpha) \equiv 1$ on $\widehat{X}$. In particular,

$$
0=\widehat{g}\left(z_{0}, w_{0}\right)\left(\widehat{f}\left(z_{0}, w_{0}\right)-\alpha\right)=1,
$$

which is a contradiction. Hence $|\widehat{f}|_{\widehat{X}} \leq|f|_{X}$. The opposite inequality is trivial since $X \subset \widehat{X}$ (see, for example, [6]).

Step 2: Proof of inequality (4.8).

Fix now $\left(z_{0}, w_{0}\right) \in \widehat{X}$. For every $\eta \in B$, we have

$$
|f(\zeta, \eta)| \leq|f|_{A \times B}, \quad \zeta \in A, \quad|f(z, \eta)| \leq|f|_{X}, \quad z \in D .
$$

Therefore, the Two-Constant Theorem (Theorem 2.2) implies that

$$
|f(z, \eta)| \leq|f|_{A \times B}^{1-\omega(z, A, D)}|f|_{X}^{\omega(z, A, D)}, \quad z \in D, \eta \in B .
$$


Consider the function $\widehat{f}\left(z_{0}, \cdot\right) \in \mathcal{O}\left(G_{z_{0}}\right)$, where

$$
G_{z_{0}}:=\left\{w \in G: \omega(w, B, G)<1-\omega\left(z_{0}, A, D\right)\right\} .
$$

Observe that $\left|\widehat{f}\left(z_{0}, \cdot\right)\right|_{G_{z_{0}}} \leq|f|_{X}$ and

$$
\omega\left(w, B, G_{z_{0}}\right)=\omega(w, B, G) /\left(1-\omega\left(z_{0}, A, D\right)\right) .
$$

Consequently, using (4.10) and applying the Two-Constant Theorem to the function $\widehat{f}\left(z_{0}, \cdot\right)$, we obtain $(4.8)$ for $\left(z_{0}, w_{0}\right)$.

Theorem 4.3. Let $D \subset \mathbb{C}^{n}$ and $G \subset \mathbb{C}^{m}$ be domains and let $A$ (resp. B) be an open subset of $\partial D$ (resp. $\partial G)$. Suppose in addition that $D$ (resp. $G)$ is locally $\mathcal{C}^{2}$ smooth on $A$ (resp. B) and $D$ is pseudoconvex. Put $X:=$ $\mathbb{X}(A, B ; D, G), \widehat{X}:=\widehat{\mathbb{X}}(A, B ; D, G)$ and $\widehat{X}^{\mathrm{o}}:=\widehat{\mathbb{X}}^{\mathrm{o}}(A, B ; D, G)$. Then for any $f \in \mathcal{C}(\widehat{X}) \cap \mathcal{O}\left(\widehat{X}^{\mathrm{o}}\right)$,

$$
|f(z, w)| \leq|f|_{A \times B}^{1-\omega(z, A, D)-\omega(w, B, G)}|f|_{X}^{\omega(z, A, D)+\omega(w, B, G)}, \quad(z, w) \in \widehat{X} .
$$

Proof. Fix $\left(z_{0}, w_{0}\right) \in \widehat{X}, \varepsilon>0$ and $\delta>0$. By Proposition 3.5 one may find an open set $T_{\delta} \subset D$ such that

$$
\omega(z, A, D)-\delta \leq \omega\left(z, T_{\delta}, D\right) \leq \omega(z, A, D), \quad z \in D .
$$

By Proposition 3.3 there is a (not necessarily pseudoconvex) bounded subdomain $G_{\delta}$ of $G$ such that $\bar{G}_{\delta} \Subset G \cup B, G_{\delta}$ is locally $\mathcal{C}^{2}$ smooth on the open subset $\partial G_{\delta} \cap B$ of $B$ and

$$
0 \leq \omega\left(w_{0}, \partial G_{\delta} \cap B, G_{\delta}\right)-\omega\left(w_{0}, B, G\right)<\delta .
$$

Since $f \in \mathcal{C}(\widehat{X})$, there is an open subset $A_{\delta}$ of $T_{\delta}$ such that $A \cup A_{\delta}$ is an open neighborhood of $A$ in $A \cup D$ and

$$
|f(z, w)| \leq|f|_{X}+\varepsilon, \quad z \in A_{\delta}, w \in G_{\delta} .
$$

It is also clear from (4.12) and the above properties of $A_{\delta}$ that

$$
\omega\left(z, A_{\delta}, D\right)-\delta \leq \omega\left(z, T_{\delta}, D\right) \leq \omega\left(z, A_{\delta}, D\right), \quad z \in D .
$$

Let $D_{\delta}$ be a strongly pseudoconvex subdomain of $D$ such that $D_{\delta} \Subset D$ and

$$
0 \leq \omega\left(z_{0}, A_{\delta} \cap D_{\delta}, D_{\delta}\right)-\omega\left(z_{0}, A_{\delta}, D\right)<\delta .
$$

Since $G_{\delta}$ is locally $\mathcal{C}^{2}$ smooth on the open subset $\partial G_{\delta} \cap B$ of $B$ and $f \in$ $\mathcal{C}(\widehat{X})$, one may find an open subset $B_{\delta}$ of $G_{\delta}$ such that $B \cup B_{\delta}$ is an open neighborhood of $\partial G_{\delta} \cap B$ in $\left(\partial G_{\delta} \cap B\right) \cup G_{\delta}$ and

$$
|f(z, w)| \leq|f|_{X}+\varepsilon, \quad z \in D_{\delta}, w \in B_{\delta} .
$$


By taking the intersection of $B_{\delta}$ with the open level set given by Proposition 3.5 for the open set $G_{\delta}$, one may assume that

$$
\begin{aligned}
\omega\left(w, \partial G_{\delta} \cap B, G_{\delta}\right)-\delta & \leq \omega\left(w, B_{\delta}, G_{\delta}\right) \\
& \leq \omega\left(w, \partial G_{\delta} \cap B, G_{\delta}\right), \quad w \in G_{\delta} .
\end{aligned}
$$

Consider the crosses

$$
X_{\delta}:=\mathbb{X}\left(A_{\delta} \cap D_{\delta}, B_{\delta} ; D_{\delta}, G_{\delta}\right), \quad \widehat{X}_{\delta}:=\widehat{\mathbb{X}}\left(A_{\delta} \cap D_{\delta}, B_{\delta} ; D_{\delta}, G_{\delta}\right) .
$$

By Theorem 1 , there is an $f_{\delta} \in \mathcal{O}\left(\widehat{X}_{\delta}\right)$ such that $f_{\delta}=f$ on $X_{\delta}$.

If one chooses $\delta$ such that $0<10 \delta<1-\omega\left(z_{0}, A, D\right)-\omega\left(w_{0}, B, G\right)$, then it follows from (4.12), (4.13), (4.15), (4.16) and (4.18) that

$$
\begin{aligned}
&\left(z_{0}, w_{0}\right) \in\left\{(z, w) \in D_{\delta} \times H_{\delta}:\right. \\
&\left.\omega\left(z, A_{\delta} \cap D_{\delta}, D_{\delta}\right)+\omega\left(w, B_{\delta}, H_{\delta}\right)<1-5 \delta\right\} \subset \widehat{X}_{\delta},
\end{aligned}
$$

where $H_{\delta}$ is the connected component of $G_{\delta}$ containing $w_{0}$.

We recall that $f_{\delta}=f$ on $X_{\delta}$. Therefore, $f\left(z_{0}, w_{0}\right)=f_{\delta}\left(z_{0}, w_{0}\right)$. Consequently, from Theorem 4.2, (4.14) and (4.17) we deduce that $\left|f\left(z_{0}, w_{0}\right)\right| \leq$ $|f|_{X}+\varepsilon$. Since $\varepsilon>0$ and $\left(z_{0}, w_{0}\right) \in \widehat{X}$ are arbitrary, it follows that $|f|_{\widehat{X}} \leq|f|$. The opposite inequality is trivial as $X \subset \widehat{X}$ by Proposition 3.6(3). Thus we have shown that $|f|_{\widehat{X}}=|f|$.

Therefore, arguing as in Step 2 of Theorem 4.2 and applying the second identity of Proposition 3.8(1), we obtain (4.11).

5. Proof of the Main Theorem for $N=2$. In this section we simplify the notation and rephrase the Main Theorem for the case $N=2$ as follows.

Theorem 5.1. Let $D \subset \mathbb{C}^{n}$ and $G \subset \mathbb{C}^{m}$ be pseudoconvex domains and let $A$ (resp. B) be an open subset of $\partial D$ (resp. $\partial G)$. Suppose in addition that $D$ (resp. $G)$ is locally $\mathcal{C}^{2}$ smooth on $A$ (resp. B). Put $X:=$ $\mathbb{X}(A, B ; D, G), X^{\mathrm{o}}:=\mathbb{X}^{\mathrm{o}}(A, B ; D, G), \widehat{X}:=\widehat{\mathbb{X}}(A, B ; D, G)$ and $\widehat{X}^{\mathrm{o}}:=$ $\widehat{\mathbb{X}}^{\mathrm{o}}(A, B ; D, G)$. Then for any $f \in \mathcal{C}(X) \cap \mathcal{O}_{\mathrm{s}}\left(X^{\mathrm{o}}\right)$, there is a unique $\widehat{f} \in$ $\mathcal{C}(\widehat{X}) \cap \mathcal{O}\left(\widehat{X}^{\circ}\right)$ such that $\widehat{f}=f$ on $X$. Moreover,

$$
|\widehat{f}(z, w)| \leq|f|_{A \times B}^{1-\omega(z, A, D)-\omega(w, B, G)}|f|_{X}^{\omega(z, A, D)+\omega(w, B, G)}, \quad(z, w) \in \widehat{X} .
$$

Proof. We proceed in several steps. First observe that by Theorem 2.1 and Proposition 3.6(3), the function $\widehat{f}$ is uniquely determined (if it exists).

SteP 1: Reduction to the case where $D$ and $G$ are bounded pseudoconvex domains.

Proof of Step 1. Fix any sequences $\left(D_{k}\right)_{k=1}^{\infty}\left(\right.$ resp. $\left.\left(G_{k}\right)_{k=1}^{\infty}\right)$ of bounded pseudoconvex subdomains of $D$ (resp. $G$ ) such that $\left(D_{k}\right)_{k=1}^{\infty}$ and $\left(A_{k}\right)_{k=1}^{\infty}$ (resp. $\left(G_{k}\right)_{k=1}^{\infty}$ and $\left.\left(B_{k}\right)_{k=1}^{\infty}\right)$ satisfy (i)-(iii) of Proposition 3.3, where $A_{k}:=$ 
$A \cap \partial D_{k}$ and $G_{k}:=B \cap \partial G_{k}$. Let

$$
X_{k}:=\mathbb{X}\left(A_{k}, B_{k} ; D_{k}, G_{k}\right) \subset X
$$

and note that $\widehat{X_{k}} \nearrow \widehat{X}$ by Proposition 3.3.

Let $f \in \mathcal{C}(X) \cap \mathcal{O}_{\mathrm{s}}\left(X^{\mathrm{o}}\right)$. Clearly, $f \in \mathcal{C}\left(X_{k}\right)$. Therefore, by the reduction assumption, for each $k$ there exists an $\widehat{f}_{k} \in \mathcal{C}\left(\widehat{X}_{k}\right) \cap \mathcal{O}\left(\widehat{X}_{k}^{o}\right)$ with $\widehat{f}_{k}=f$ on $X_{k}$. By Theorem 2.1 and Proposition 3.6, $\widehat{f}_{k+1}=\widehat{f}_{k}$ on $\widehat{X}_{k}$. Therefore, gluing the $\widehat{f}_{k}$ 's, we obtain an $\widehat{f} \in \mathcal{C}(\widehat{X}) \cap \mathcal{O}\left(\widehat{X}^{\circ}\right)$ with $\widehat{f}=f$ on $X$. To reduce estimate (5.1) to the case where $D$ and $G$ are bounded pseudoconvex domains, we proceed in the same way as above. This completes Step 1.

From now on we assume that the hypothesis of Step 1 is fulfilled.

We introduce a new terminology. A subset $\mathcal{A}$ of an open subset $A$ of $\partial D$ is said to be a ball in $A$ with center $\zeta$ and radius $r$ if $\mathcal{A}=B(\zeta, r) \cap A$ for a point $\zeta \in A$ and a positive number $r$ satisfying $2 r<\operatorname{dist}(\zeta, \partial A)$. Moreover, for a ball $\mathcal{A}$ in $A$ and a number $0<\lambda \leq 2, \lambda \mathcal{A}$ denotes the open set $B(\zeta, \lambda r) \cap \partial D$.

STEP 2: Keep the hypothesis of Theorem 5.1 and assume in addition that $G$ is a Jordan planar simply connected domain. Then the following local version of Theorem 5.1 holds:

For any $P \in A$, there is a ball $\mathcal{A}$ in $A$ with center $P$ such that for any $f \in \mathcal{C}(X) \cap \mathcal{O}_{\mathrm{s}}\left(X^{\mathrm{o}}\right)$, there is a unique $\widehat{f} \in \mathcal{C}\left(\widehat{X}_{\mathcal{A}}\right) \cap \mathcal{O}\left(\widehat{X}_{\mathcal{A}}^{\mathrm{o}}\right)$ with $\widehat{f}=f$ on $X_{\mathcal{A}}$, where

$$
X_{\mathcal{A}}:=\mathbb{X}(\mathcal{A}, B ; D, G), \quad \widehat{X}_{\mathcal{A}}:=\widehat{\mathbb{X}}(\mathcal{A}, B ; D, G), \quad \widehat{X}_{\mathcal{A}}^{\mathrm{o}}:=\widehat{\mathbb{X}}^{\mathrm{o}}(\mathcal{A}, B ; D, G) .
$$

Proof of Step 2. First, we apply Proposition 2.5 to the domain $D$ which is locally $\mathcal{C}^{2}$ smooth on an open neighborhood of $P$ in $\partial D$. Consequently, there is an open neighborhood $U$ of $P$ satisfying (2.2) such that Proposition 2.5 is applicable there. Below, $U, U_{1}, U_{3}, \pi^{\mathbb{C}}, \pi, V$ and $V_{Q}$ have the same meaning as in Proposition 2.5. Now we can fix a ball $\mathcal{A}$ of $A$,

$$
\mathcal{A}:=A \cap B(P, r),
$$

where the radius $r$ is so small that $2 \mathcal{A} \Subset A, 2 \mathcal{A} \Subset \partial(U \cap D) \cap \partial V, 2 \mathcal{A} \Subset U_{3}$ and $\pi^{\mathbb{C}}(2 \mathcal{A}) \Subset U_{1}$.

For any $\delta$ small enough, by Proposition 3.5 we may find an open subset $T_{\delta}$ of $U \cap D$ such that

$$
\begin{aligned}
& \omega(z, \mathcal{A}, D)-\delta \leq \omega\left(z, T_{\delta}, D\right) \leq \omega(z, \mathcal{A}, D), \quad z \in D, \\
& \sup _{T_{\delta}} \operatorname{dist}(\cdot, \mathcal{A})<\delta, \quad \pi^{\mathbb{C}}\left(T_{\delta}\right) \Subset U_{1} .
\end{aligned}
$$

A geometric argument based on Proposition 2.5 and definition (5.2) shows that one may find $\delta_{0}>0$ small enough such that for any $z \in D \cup A$ with $\operatorname{dist}(z, 2 \mathcal{A})<\delta_{0}$, we have $z \in U_{3}$ and there is a unique $Q_{z} \in U_{1}$ such that 
$z \in V_{Q_{z}}$. Moreover, by Proposition 2.5(4) we have

$$
\operatorname{dist}\left(z, \partial V_{Q_{z}} \cap \frac{3}{2} \mathcal{A}\right) \leq C_{1} \operatorname{dist}(z, \mathcal{A})
$$

for any $z \in D \cup A$ with $\operatorname{dist}(z, \mathcal{A})<\delta_{0}$. On the other hand, combining Corollary 2.4 and Propositions 2.5 and 3.1, we get

$$
\omega\left(z, \partial V_{Q_{z}} \cap 2 \mathcal{A}, V_{Q_{z}}\right) \leq C_{2} \operatorname{dist}\left(z, \partial V_{Q_{z}} \cap \frac{3}{2} \mathcal{A}\right),
$$

where $C_{1}, C_{2}$ are constants independent of $z \in D \cup A$ with $\operatorname{dist}(z, \mathcal{A})<\delta_{0}$.

For each $Q \in \pi^{\mathbb{C}}(2 \mathcal{A}$ ), we apply Gonchar's Theorem (Theorem 2) to $f \in \mathcal{C}\left(\mathbb{X}\left(\partial V_{Q} \cap 2 \mathcal{A}, B ; V_{Q}, G\right)\right) \cap \mathcal{O}_{\mathrm{s}}\left(\mathbb{X}^{\circ}\left(\partial V_{Q} \cap 2 \mathcal{A}, B ; V_{Q}, G\right)\right)$ to obtain $\tilde{f}_{Q} \in$ $\mathcal{C}\left(\widehat{\mathbb{X}}\left(\partial V_{Q} \cap 2 \mathcal{A}, B ; V_{Q}, G\right)\right) \cap \mathcal{O}\left(\widehat{\mathbb{X}}^{\circ}\left(\partial V_{Q} \cap 2 \mathcal{A}, B ; V_{Q}, G\right)\right)$ such that $\widetilde{f}_{Q}=f$ on $\mathbb{X}\left(\partial V_{Q} \cap 2 \mathcal{A}, B ; V_{Q}, G\right)$.

Gluing the family $\left(\widetilde{f}_{Q}\right)_{Q \in \pi^{\mathbb{C}}(2 \mathcal{A})}$, we obtain an extension function $\tilde{f}$ defined on the set

$$
\begin{aligned}
\widetilde{X}_{\mathcal{A}}:=\left\{(z, w) \in(D \cup 2 \mathcal{A}) \times(G \cup B): \exists Q \in \pi^{\mathbb{C}}(2 \mathcal{A}), z \in V_{Q}\right. \\
\text { and } \left.\omega\left(z, \partial V_{Q_{z}} \cap 2 \mathcal{A}, V_{Q_{z}}\right)+\omega(w, B, G)<1\right\},
\end{aligned}
$$

which is not necessarily open; moreover

$$
\widetilde{f}=f \quad \text { on } \mathbb{X}\left(\mathcal{A}, B ; T_{\delta_{0}}, G\right) .
$$

By (5.3)-(5.5) we obtain a $\delta_{0}$ small enough such that for $0<\delta<\delta_{0}$,

$$
\omega\left(z, \partial V_{Q_{z}} \cap 2 \mathcal{A}, V_{Q_{z}}\right) \leq C_{1} C_{2} \operatorname{dist}(z, \mathcal{A})<C_{1} C_{2} \delta<1, \quad z \in T_{\delta} .
$$

Therefore, by (5.6), (5.8) and Theorem 2 for $0<\delta<\delta_{1}:=\min \left\{\delta_{0}, 1 / 2 C_{1} C_{2}\right\}$ and $z \in T_{\delta}, \widetilde{f}(z, \cdot)$ is holomorphic on the open set

$$
G_{\delta}:=\left\{w \in G: \omega(w, B, G)<1-2 C_{1} C_{2} \delta\right\} .
$$

We need the following

Lemma 5.2. For any $\left(\zeta_{0}, w_{0}\right) \in \overline{\mathcal{A}} \times(G \cup B)$, there are an open neighborhood $\mathcal{U}$ of $\zeta_{0}$ in $D \cup A$ and an open neighborhood $\mathcal{V}$ of $w_{0}$ in $G \cup B$ such that $\mathcal{U} \times \mathcal{V} \subset \widetilde{X}_{\mathcal{A}}$ and $|\widetilde{f}|_{\mathcal{U} \times \mathcal{V}}<\infty$.

Proof of Lemma 5.2. Fix $\left(\zeta_{0}, w_{0}\right) \in \overline{\mathcal{A}} \times(G \cup B)$. Let

$$
\varepsilon:=\frac{1-\omega\left(w_{0}, B, G\right)}{3}
$$

and choose an open neighborhood $\mathcal{V}$ of $w_{0}$ in $G \cup B$ such that

$$
\omega(w, B, G)<\omega\left(w_{0}, B, G\right)+\varepsilon, \quad w \in \mathcal{V} .
$$


Moreover, by (5.4) and (5.5) choose an open neighborhood $\mathcal{U}$ of $\zeta_{0}$ in $D \cup A$ such that

$$
\omega\left(z, \partial V_{Q_{z}} \cap 2 \mathcal{A}, V_{Q_{z}}\right)<\varepsilon, \quad z \in \mathcal{U} .
$$

Next, by Proposition 3.3, we may find a subdomain $G^{\varepsilon}$ of $G$ such that $w_{0} \in G^{\varepsilon}, \overline{G^{\varepsilon}} \subset G \cup B$ and $G^{\varepsilon}$ is locally $\mathcal{C}^{2}$ smooth on the open subset $B_{\varepsilon}:=\partial G \cap \partial G^{\varepsilon}$ of $B, B_{\varepsilon} \Subset B$ and

$$
\omega\left(w_{0}, B, G\right) \leq \omega\left(w_{0}, B_{\varepsilon}, G^{\varepsilon}\right)<\omega\left(w_{0}, B, G\right)+\varepsilon .
$$

By shrinking $\mathcal{V}$ if necessary, we may assume that

$$
\omega\left(w, B_{\varepsilon}, G^{\varepsilon}\right)<\omega\left(w_{0}, B_{\varepsilon}, G^{\varepsilon}\right)+\varepsilon, \quad w \in \mathcal{V} .
$$

Since $f \in \mathcal{C}(X)$ and $2 \mathcal{A} \Subset A$, by shrinking $\mathcal{U}$ if necessary, we may find a constant $M$ such that

$$
|\widetilde{f}|_{2 \mathcal{A} \times G^{\varepsilon}}<M, \quad|\widetilde{f}|_{\mathcal{U} \times B_{\varepsilon}}<M .
$$

Consequently, for each $Q \in \pi^{\mathbb{C}}(2 \mathcal{A})$ we can apply Gonchar's Theorem (Theorem 2) to $f \in \mathcal{C}\left(\mathbb{X}\left(\partial V_{Q} \cap 2 \mathcal{A}, B_{\varepsilon} ; V_{Q}, G^{\varepsilon}\right)\right) \cap \mathcal{O}_{\mathrm{s}}\left(\mathbb{X}^{\circ}\left(\partial V_{Q} \cap 2 \mathcal{A}, B_{\varepsilon} ; V_{Q}, G^{\varepsilon}\right)\right)$ to obtain the inequality $|\widetilde{f}|<M$ on

$$
\begin{array}{r}
\widetilde{X}_{\mathcal{A}}^{\varepsilon}:=\left\{(z, w) \in(D \cup 2 \mathcal{A}) \times\left(G^{\varepsilon} \cup B_{\varepsilon}\right): \exists Q \in \pi^{\mathbb{C}}(2 \mathcal{A}), z \in V_{Q}\right. \\
\text { and } \left.\omega\left(z, \partial V_{Q_{z}} \cap 2 \mathcal{A}, V_{Q_{z}}\right)+\omega\left(w, B_{\varepsilon}, G^{\varepsilon}\right)<1\right\},
\end{array}
$$

On the other hand, using (5.6) and (5.11)-(5.14), we see that

$$
\mathcal{U} \times \mathcal{V} \subset \widetilde{X}_{\mathcal{A}}^{\varepsilon} \subset \widetilde{X}_{\mathcal{A}}
$$

Hence $|\widetilde{f}|_{\mathcal{U} \times \mathcal{V}}<M$, which completes the proof of the lemma.

Now for any $0<\delta<\delta_{1}$, we can apply Theorem 4.1 to the function

$$
\tilde{f} \in \mathcal{C}_{\mathbf{S}}\left(\mathbb{X}\left(T_{\delta}, B ; D, G_{\delta}\right)\right) \cap \mathcal{O}_{\mathbf{s}}\left(\mathbb{X}^{\mathrm{o}}\left(T_{\delta}, B ; D, G_{\delta}\right)\right)
$$

to obtain $\widehat{f}_{\delta} \in \mathcal{C}\left(\widehat{\mathbb{X}}\left(T_{\delta}, B ; D, G_{\delta}\right)\right) \cap \mathcal{O}\left(\widehat{\mathbb{X}}^{\circ}\left(T_{\delta}, B ; D, G_{\delta}\right)\right)$ such that

$$
\widehat{f_{\delta}}=\widetilde{f} \quad \text { on } \mathbb{X}\left(T_{\delta}, B ; D, G_{\delta}\right) .
$$

We are now in a position to define the desired extension function $\widehat{f}$. Indeed, one glues $\left(\widehat{f}_{\delta}\right)_{0<\delta<\delta_{1}}$ together to obtain $\widehat{f}$ in the following way:

$$
\widehat{f}:=\lim _{\delta \rightarrow 0} \widehat{f}_{\delta} \quad \text { on } \widehat{\mathbb{X}}(\mathcal{A}, B ; D, G) \backslash(\mathcal{A} \times(G \cup B)) .
$$

One now checks that the limit (5.17) exists and has all the required properties. This is an immediate consequence of

Lemma 5.3. For any $(z, w) \in \widehat{\mathbb{X}}(\mathcal{A}, B ; D, G) \backslash(\mathcal{A} \times(G \cup B))$ let $\delta_{z, w}$ be the unique positive number $\delta$ which satisfies 


$$
\delta+\frac{2 C_{1} C_{2} \delta}{1-\delta}=1-\omega(z, \mathcal{A}, D)-\omega(w, B, G)
$$

Then $f(z, w)=\widehat{f}_{\delta}(z, w)$ for all $0<\delta<\delta_{z, w}$.

Proof of Lemma 5.3. Fix $\left(z_{0}, w_{0}\right) \in \widehat{\mathbb{X}}(\mathcal{A}, B ; D, G) \backslash(\mathcal{A} \times(G \cup B))$. Then by (5.3), (5.18) and the second identity of Proposition 3.8(1), for all $0<$ $\delta<\delta_{z_{0}, w_{0}}$

$$
\left(z_{0}, w_{0}\right) \in \widehat{\mathbb{X}}\left(T_{\delta}, B ; D, G_{\delta}\right) .
$$

Therefore, for any $0<\delta^{\prime}<\delta<\delta_{z_{0}, w_{0}},\left(z_{0}, w_{0}\right)$ is in the set

$$
\widehat{\mathbb{X}}\left(T_{\delta^{\prime}}, B ; D, G_{\delta^{\prime}}\right) \cap \widehat{\mathbb{X}}\left(T_{\delta}, B ; D, G_{\delta}\right) .
$$

Let $H_{\delta}$ be the connected component of $G_{\delta}$ containing $w_{0}$. Let $B_{\delta}$ be the largest open subset of $B$ such that $H_{\delta}$ is locally $\mathcal{C}^{2}$ smooth on $B_{\delta}$. By Proposition 3.8(2), $B_{\delta}$ is nonempty and $\omega\left(w, B_{\delta}, H_{\delta}\right)=\omega\left(w, B, G_{\delta}\right)$ for $w \in H_{\delta}$. On the other hand, using (5.3) and the inclusion $G_{\delta} \subset G_{\delta^{\prime}}$, we see that the set (5.19) contains

$$
\left\{(z, w) \in D \times H_{\delta}: \omega\left(z, T_{\delta}, D\right)+\omega\left(w, B_{\delta}, H_{\delta}\right)<1-\delta\right\} .
$$

By Proposition 3.7 this last open set is connected. Moreover, it contains $\left(z_{0}, w_{0}\right)$. In addition by (5.7) and (5.16), one gets

$$
\widehat{f}_{\delta^{\prime}}=\widehat{f}_{\delta}=\widetilde{f}=f \quad \text { on } T_{\delta} \times B_{\delta} .
$$

Applying Theorem 2.1, we deduce that $\widehat{f}_{\delta^{\prime}}=\widehat{f}_{\delta}$ on the domain given by (5.20). In particular $\widehat{f}_{\delta^{\prime}}\left(z_{0}, w_{0}\right)=\widehat{f}_{\delta}\left(z_{0}, w_{0}\right)$. This completes the proof.

Another consequence of Lemma 5.3 is that $\widehat{f} \in \mathcal{O}\left(\widehat{\mathbb{X}}^{\circ}(\mathcal{A}, B ; D, G)\right)$. Now we define by

$$
\widehat{f}:=f \quad \text { on } \mathcal{A} \times(G \cup B) .
$$

Thus $\widehat{f}$ is well defined on the whole $\widehat{\mathbb{X}}(\mathcal{A}, B ; D, G)$.

To complete Step 2, it remains to show that $\widehat{f} \in \mathcal{C}(\widehat{\mathbb{X}}(\mathcal{A}, B ; D, G))$ and $\widehat{f}=f$ on $\mathbb{X}(\mathcal{A}, B ; D, G)$.

First we prove that $\widehat{f}$ is continuous on $D \times B$. For this let $\left(z_{0}, \eta_{0}\right) \in D \times B$. By Proposition 3.2 there are an open neighborhood $\mathcal{U}$ of $z_{0}$ in $D$ and an open neighborhood $\mathcal{V}$ of $\zeta_{0}$ in $G \cup B$ such that

$$
\lambda:=\sup _{z \in \mathcal{U}, w \in \mathcal{V}}(\omega(z, \mathcal{A}, D)+\omega(w, B, G))<1 .
$$

Now let $\delta>0$ satisfy $\delta+2 C_{1} C_{2} \delta /(1-\delta)<1-\lambda$. Then Lemma 5.3 implies that $f=\widehat{f}_{\delta}$ on $\mathcal{U} \times \mathcal{V}$. Since from Theorem 4.1 we know that $\widehat{f}_{\delta}$ is continuous on $D \times B$, so is $\widehat{f}$ and moreover $\widehat{f}=f$ on $D \times B$. This, combined with (5.21), implies that $\widehat{f}=f$ on $\mathbb{X}(\mathcal{A}, B ; D, G)$. 
Finally, it remains to check the continuity of $\widehat{f}$ on $\mathcal{A} \times(G \cup B)$. Fix $\left(\zeta_{0}, w_{0}\right) \in \mathcal{A} \times(G \cup B)$ and $0<\varepsilon<1$. As $f \in \mathcal{C}(X)$, by Lemma 5.2 there are an open connected neighborhood $\mathcal{U}$ of $\zeta_{0}$ in $D \cup \mathcal{A}$, an open connected neighborhood $\mathcal{V}$ of $w_{0}$ in $G \cup B$ and a constant $M$ such that

$$
\begin{aligned}
\left|f\left(\zeta_{0}, w_{0}\right)-f(\zeta, w)\right| & <\varepsilon^{2}, \quad \zeta \in \mathcal{A} \cap \mathcal{U}, w \in \mathcal{V}, \\
|\widetilde{f}|_{\mathcal{U} \times \mathcal{V}} & <M / 2 .
\end{aligned}
$$

Moreover, by shrinking $\mathcal{U}$ and $\mathcal{V}$ if necessary, and applying Proposition 3.2, we may suppose that

$$
\sup _{\mathcal{U} \times \mathcal{V}}(\omega(z, \mathcal{A}, D)+\omega(w, B, G))<1 .
$$

Therefore, $\mathcal{U} \times \mathcal{V} \subset \widehat{\mathbb{X}}(\mathcal{A}, B ; D, G)$. Moreover, by Lemma 5.3 and (5.17), there is a $\delta>0$ such that $\widehat{f}=\widehat{f}_{\delta}=\widetilde{f}$ on the nonempty open set $\left(T_{\delta} \cap \mathcal{U}\right) \times \mathcal{V}$. Thus

$$
\widehat{f}=\widetilde{f} \quad \text { on } \mathcal{U} \times \mathcal{V} .
$$

By shrinking $\mathcal{U}$ if necessary, we may suppose that for all $z \in \mathcal{U}$, there is exactly one $\zeta_{z} \in \mathcal{A}$ such that $\pi\left(\zeta_{z}\right)=\pi(z)$. By Proposition 2.5(4) we have

$$
z \in V_{Q_{z}}, \quad \zeta_{z} \in \mathcal{A} \cap \partial V_{Q_{z}}, \quad \operatorname{dist}\left(z, \zeta_{z}\right) \approx \operatorname{dist}\left(z, \partial V_{Q_{z}}\right) .
$$

Therefore, we can apply the Two-Constant Theorem to the function $\widetilde{f}(\cdot, w)-$ $\widetilde{f}\left(\zeta_{z}, w\right) \in \mathcal{C}\left(\left(\partial V_{Q_{z}} \cap \mathcal{U}\right) \cup\left(V_{Q_{z}} \cap \mathcal{U}\right)\right) \cap \mathcal{O}\left(V_{Q_{z}} \cap \mathcal{U}\right)$, which is, by (5.22), bounded by $M$ for any $z \in \mathcal{U}, w \in \mathcal{V}$.

Consequently, taking (5.22) and (5.23) into account, we deduce that

$$
\left|\widehat{f}(z, w)-\widehat{f}\left(\zeta_{z}, w\right)\right|<\varepsilon^{2\left(1-\omega\left(z, \partial V_{Q_{z}} \cap \mathcal{U}, V_{Q_{z}} \cap \mathcal{U}\right)\right)} M^{\omega\left(z, \partial V_{Q_{z}} \cap \mathcal{U}, V_{Q_{z}} \cap \mathcal{U}\right)}
$$

for all $(z, w) \in \mathcal{U} \times \mathcal{V}$. Thus for $(z, w) \in(D \cup \mathcal{A}) \times(G \cup B)$ sufficiently close to $\left(\zeta_{0}, w_{0}\right)$, by Proposition 3.2 and $(5.22)$ we have

$$
\begin{aligned}
\left|\widehat{f}(z, w)-\widehat{f}\left(\zeta_{0}, w_{0}\right)\right| & \leq\left|\widehat{f}(z, w)-\widehat{f}\left(\zeta_{z}, w\right)\right|+\left|\widehat{f}\left(\zeta_{z}, w\right)-\widehat{f}\left(\zeta_{0}, w_{0}\right)\right| \\
& <\varepsilon / 2+\varepsilon / 2=\varepsilon,
\end{aligned}
$$

which proves the continuity of $\widehat{f}$ at $\left(\zeta_{0}, w_{0}\right)$.

Hence Step 2 is finished.

STEP 3: The case where $G$ is a Jordan planar simply connected domain.

Proof of Step 3. Fix a sequence $\left(A_{k}\right)_{k=1}^{\infty}$ of open subsets of $A$ such that $A_{k} \Subset A_{k+1}$ and $A_{k} \nearrow A$ as $k \nearrow \infty$. By Proposition 3.4, $\widehat{\mathbb{X}}\left(A_{k}, B ; D, G\right) \nearrow$ $\widehat{\mathbb{X}}(A, B ; D, G)$. Using a routine uniqueness argument (Theorem 2.1 and Proposition 3.6) and the gluing procedure, we are reduced to proving that for any $k$, there is an $\widehat{f}_{k} \in \mathcal{C}\left(\widehat{\mathbb{X}}\left(A_{k}, B ; D, G\right)\right) \cap \mathcal{O}\left(\widehat{\mathbb{X}}^{\circ}\left(A_{k}, B ; D, G\right)\right)$ satisfying $\widehat{f}_{k}=f$ on $\mathbb{X}\left(A_{k}, B ; D, G\right)$. 
Now fix $k \in \mathbb{N}$. First we show that one may find a $\delta_{0}>0$ with the following properties:

For any $0<\delta<\delta_{0}$, there are a finite number of open balls $\left(\mathcal{A}_{j}\right)_{j=1}^{N}$ of $A$ with radius $\delta$ ( $N$ depending on $\delta$ ) such that

(i) $A_{k} \subset \bigcup_{j=1}^{N} \mathcal{A}_{j}$;

(ii) $\bigcup_{j=1}^{N} 2 \mathcal{A}_{j} \subset A_{k+1}$, where $2 \mathcal{A}_{j}$ is the ball with the same center as $\mathcal{A}_{j}$ but with double radius;

(iii) for each $1 \leq j \leq N$, Step 2 applies to the open ball $2 \mathcal{A}_{j}$; more precisely, Step 2 provides $\widehat{f}_{j} \in \mathcal{C}\left(\widehat{X}_{2 \mathcal{A}_{j}}\right) \cap \mathcal{O}\left(\widehat{X}_{2 \mathcal{A}_{j}}^{\mathrm{o}}\right)$ satisfying $\widehat{f}_{j}=f$ on $X_{2 \mathcal{A}_{j}}$;

(iv) for any $0<\delta<\delta_{0}$ there is an open subset $T_{\delta}$ of $D$ such that

$$
\begin{aligned}
& \omega\left(z, A_{k}, D\right)-\delta \leq \omega\left(z, T_{\delta}, D\right) \leq \omega\left(z, A_{k}, D\right), \quad z \in D, \\
& \sup _{T_{\delta}} \operatorname{dist}\left(\cdot, A_{k}\right)<r,
\end{aligned}
$$

for some $0<r:=r_{\delta}<\delta$;

(v) for any $0<\delta<\delta_{0}$ and $z \in T_{\delta}$ there is a unique $\zeta_{z} \in \bigcup_{j=1}^{N} \mathcal{A}_{j}$ such that $\operatorname{dist}\left(z, \zeta_{z}\right)=\operatorname{dist}(z, \partial D)$ and for any $1 \leq j \leq N$ such that $\zeta_{z} \in \mathcal{A}_{j}$ we have

$$
\sup _{t \in\left[z, \zeta_{z}\right]} \omega\left(t, 2 \mathcal{A}_{j}, D\right)<\delta,
$$

where $\left[z, \zeta_{z}\right]$ denotes the real segment connecting $z$ to $\zeta_{z}$.

Indeed, by Step 2 and a compactness argument we see that one may find $\delta_{0}>0$ such that (i)-(iii) are satisfied.

On the other hand, using Proposition 3.2 we see that there is an $r:=r_{\delta}$ such that

$$
\omega\left(z, 2 \mathcal{A}_{j}, D\right)<\delta
$$

for all $1 \leq j \leq N$ and $z \in D$ with $\operatorname{dist}\left(z, \mathcal{A}_{j}\right)<r$.

By examining carefully the proof of Proposition 3.5, we may arrange $T_{\delta}$ in such a way that (iv) holds with the $r$ given above. It is also clear that when $r$ is sufficiently small, the first assertion of $(\mathrm{v})$ is satisfied, and the second is an immediate consequence of (5.24).

Thus we have verified properties (i) $-(\mathrm{v})$.

Next fix $0<\delta<\delta_{0}$ and put

$$
G_{\delta}:=\{w \in G: \omega(w, B, G)<1-2 \delta\} .
$$

We define a new function $\tilde{f}$ on $\left(T_{\delta} \cup A_{k}\right) \times G_{\delta}$ as follows. For any $(z, w) \in$ $\left(T_{\delta} \cup A_{k}\right) \times G_{\delta}$ let

$$
\widetilde{f}(z, w):=\widehat{f}_{j}(z, w)
$$

for any $1 \leq j \leq N$ such that $\zeta_{z} \in \mathcal{A}_{j}$ (see (v) above). 
First one checks that $\tilde{f}$ is well defined. Indeed, in view of (iv)-(v) and (5.25), for any $(z, w) \in\left(T_{\delta} \cup A_{k}\right) \times G_{\delta}$ there is $j$ such that $\zeta_{z} \in \mathcal{A}_{j}$ and $(t, w) \in$ $\widehat{X}_{2 \mathcal{A}_{j}}$ for $t \in\left[z, \zeta_{z}\right]$. Suppose that also $\zeta_{z} \in \mathcal{A}_{l}$. Observe that $\widehat{f}_{j}=\widehat{f}_{l}=f$ on $\left(\mathcal{A}_{j} \cap \mathcal{A}_{l}\right) \times G$. Therefore, Theorem 2.1 and Proposition 3.6 yield $\widehat{f}_{j}=\widehat{f}_{l}$ on the connected component of $\widehat{X}_{2 \mathcal{A}_{j}} \cap \widehat{X}_{2 \mathcal{A}_{l}}$ which is locally $\mathcal{C}^{2}$ smooth on $\left(\mathcal{A}_{j} \cap \mathcal{A}_{l}\right) \times G_{\delta}$. However, we have already shown in the previous paragraph that $(t, w) \in \widehat{X}_{2 \mathcal{A}_{j}} \cap \widehat{X}_{2 \mathcal{A}_{l}}$ for $t \in\left[z, \zeta_{z}\right]$, and clearly $\left(\zeta_{z}, w\right) \in\left(\mathcal{A}_{j} \cap \mathcal{A}_{l}\right) \times G$. Consequently, the above mentioned connected component contains $(z, w)$. Thus $\widehat{f}_{j}(z, w)=\widehat{f}_{l}(z, w)$, and hence $\widetilde{f}$ is well defined.

In view of (5.26), it is also clear that

$$
\widetilde{f} \in \mathcal{C}\left(\left(T_{\delta} \cup A_{k}\right) \times G_{\delta}\right) \cap \mathcal{O}\left(T_{\delta} \times G_{\delta}\right) .
$$

Let $\widetilde{f}_{\delta}$ be the trace of $\widetilde{f}$ on $\mathbb{X}\left(T_{\delta}, B ; D, G_{\delta}\right)$. Applying Theorem 4.1 to $\widetilde{f}_{\delta} \in$ $\mathcal{C}\left(\mathbb{X}\left(T_{\delta}, B ; D, G_{\delta}\right)\right) \cap \mathcal{O}\left(\mathbb{X}^{\mathrm{o}}\left(T_{\delta}, B ; D, G_{\delta}\right)\right)$, we obtain

$$
\widehat{f}_{\delta} \in \mathcal{C}\left(\widehat{\mathbb{X}}\left(T_{\delta}, B ; D, G_{\delta}\right)\right) \cap \mathcal{O}\left(\widehat{\mathbb{X}}^{\circ}\left(T_{\delta}, B ; D, G_{\delta}\right)\right)
$$

satisfying $\widehat{f}_{\delta}=f$ on $D \times B$.

Finally, one proceeds as at the end of Step 2. Observe that Lemma 5.3 is still valid in the present context. As in formula (5.17), one may glue $\left(\widehat{f_{\delta}}\right)_{0<\delta<\delta_{0}}$ to obtain an extension function $\widehat{f}:=\lim _{\delta \rightarrow 0} \widehat{f_{\delta}}$ which is holomorphic on $\widehat{X}^{\mathrm{o}}$ and continuous on $D \times B$.

Since $\left.\widetilde{f} \in \mathcal{C}\left(\left(T_{\delta} \cup A_{k}\right) \times G_{\delta}\right)\right)$ for $0<\delta<\delta_{0}$, Lemma 5.3 in the present context also shows that $\widehat{f} \in \mathcal{C}(\widehat{\mathbb{X}}(A, B ; D, G))$.

Hence Step 3 is complete.

STEP 4: Under the hypothesis of Theorem 5.1, the following local version of that theorem holds:

For any $P \in B$, there is a ball $\mathcal{B}$ in $B$ with center $P$ such that for any $f \in \mathcal{C}(X) \cap \mathcal{O}_{\mathrm{s}}\left(X^{\mathrm{o}}\right)$, there is a unique $\widehat{f} \in \mathcal{C}\left(\widehat{X}_{\mathcal{B}}\right) \cap \mathcal{O}\left(\widehat{X}_{\mathcal{B}}^{\mathrm{o}}\right)$ with $\widehat{f}=f$ on $X_{\mathcal{B}}$, where

$$
X_{\mathcal{B}}:=\mathbb{X}(A, \mathcal{B} ; D, G), \quad \widehat{X}_{\mathcal{B}}:=\widehat{\mathbb{X}}(A, \mathcal{B} ; D, G), \quad \widehat{X}_{\mathcal{B}}^{\mathrm{o}}:=\widehat{\mathbb{X}}^{\circ}(A, \mathcal{B} ; D, G) .
$$

Proof of Step 4. Using Step 3, we proceed in exactly the same way as we did in Step 2 using Theorem 2. Therefore we only briefly outline the proof.

First we apply Proposition 2.5 to the domain $G$ which is locally $\mathcal{C}^{2}$ smooth on an open neighborhood of $P \in \partial G$. Consequently, there is an open neighborhood $U$ of $P$ satisfying (2.2) such that Proposition 2.5 is applicable there. Below, $U, U_{1}, \pi^{\mathbb{C}}, V$ and $V_{Q}$ have the same meaning as in Proposition 2.5. Now we can fix a ball $\mathcal{B}:=B \cap B(P, r)$, with $r$ so small that $2 \mathcal{B} \Subset B$ etc. 
Arguing as in (5.2)-(5.3) we can choose $\delta_{0}>0$ such that for any $0<$ $\delta<\delta_{0}$ there is an open subset $S_{\delta}$ of $G$ satisfying

$$
\begin{aligned}
& \omega(w, \mathcal{B}, G)-\delta \leq \omega\left(w, S_{\delta}, G\right) \leq \omega(w, \mathcal{B}, G), \quad w \in G, \\
& \sup _{S_{\delta}} \operatorname{dist}(\cdot, \mathcal{B})<\delta .
\end{aligned}
$$

Arguing as in (5.4)-(5.8), there is a constant $C_{3}$ such that

$$
\omega\left(w, \partial V_{Q_{w}} \cap 2 \mathcal{B}, V_{Q_{w}}\right) \leq C_{3} \operatorname{dist}(w, \mathcal{B}) \leq C_{3} \delta
$$

for $0<\delta<\delta_{0}, w \in S_{\delta}$ and $Q_{w}:=\pi^{\mathbb{C}}(w)$.

Lemma 5.2 is still valid in the present context, with obvious changes in notation. There is only one important difference between Step 2 and the present step. In Step 2 we applied Gonchar's Theorem to (5.15), while now we appeal to Theorem 4.3.

Lemma 5.3 is also valid in the present context, with obvious changes in notation.

For each $Q \in \pi^{\mathbb{C}}(2 \mathcal{B})$, we apply the result of Step 3 to $f \in \mathcal{C}\left(\mathbb{X}\left(A, \partial V_{Q} \cap\right.\right.$ $\left.\left.2 \mathcal{B} ; D, V_{Q}\right)\right) \cap \mathcal{O}_{\mathrm{s}}\left(\mathbb{X}^{\mathrm{O}}\left(A, \partial V_{Q} \cap 2 \mathcal{B} ; D, V_{Q}\right)\right)$ to obtain $\widehat{f}_{Q} \in \mathcal{C}\left(\widehat{\mathbb{X}}\left(A, \partial V_{Q} \cap\right.\right.$ $\left.\left.2 \mathcal{B} ; D, V_{Q}\right)\right) \cap \mathcal{O}\left(\widehat{\mathbb{X}}^{\circ}\left(A, \partial V_{Q} \cap 2 \mathcal{B} ; D, V_{Q}\right)\right)$ such that $\widehat{f}_{Q}=f$ on $\mathbb{X}\left(A, \partial V_{Q} \cap\right.$ $\left.2 \mathcal{B} ; D, V_{Q}\right)$.

Gluing the family $\left(\widehat{f}_{Q}\right)_{Q \in \pi^{\mathbb{C}}(2 \mathcal{B})}$, we obtain an extension function $\tilde{f}$ defined on

$$
\begin{aligned}
& \left\{(z, w) \in D \times G: \exists Q \in \pi^{\mathbb{C}}(2 \mathcal{B}), w \in V_{Q}\right. \text { and } \\
& \left.\qquad(z, A, D)+\omega\left(w, \partial V_{Q} \cap 2 \mathcal{B}, V_{Q}\right)<1\right\} .
\end{aligned}
$$

For $0<\delta<\delta_{0}$ put

$$
D_{\delta}:=\left\{\omega(z, A, D)<1-2 C_{3} \delta\right\} .
$$

As in Step 2, taking (5.27)-(5.30) into account we see that

$$
\tilde{f} \in \mathcal{C}_{\mathrm{S}}\left(\mathbb{X}\left(A, S_{\delta} ; D_{\delta}, G\right)\right) \cap \mathcal{O}_{\mathrm{s}}\left(\mathbb{X}^{\mathrm{O}}\left(A, S_{\delta} ; D_{\delta}, G\right)\right) .
$$

Therefore, we can apply Theorem 4.1 to obtain an extension function

$$
\widehat{f_{\delta}} \in \mathcal{C}\left(\widehat{\mathbb{X}}\left(A, S_{\delta} ; D_{\delta}, G\right)\right) \cap \mathcal{O}\left(\widehat{\mathbb{X}}^{\circ}\left(A, S_{\delta} ; D_{\delta}, G\right)\right) .
$$

Using $(5.17)$ we may glue $\left(\widehat{f}_{\delta}\right)_{0<\delta<\delta_{0}}$ together to obtain the desired $\widehat{f}$. The rest of the proof follows Step 2, making use of the Two-Constant Theorem and Lemmas 5.2 and 5.3. This finishes Step 4.

STEP 5: The general case.

The argument used to go from Step 2 to Step 3 also enables us to go from Step 4 to Step 5. Consequently, there is an $\widehat{f} \in \mathcal{C}(\widehat{X}) \cap \mathcal{O}_{\mathrm{s}}\left(\widehat{X}^{\mathrm{o}}\right)$ such that $\widehat{f}=f$ on $X$. It is also clear that $\widehat{f}$ is uniquely determined. Finally, estimate (5.1) follows immediately from Theorem 4.3.

This completes the last step of the proof. 
6. Proof of the Main Theorem and concluding remarks. In order to prove the Main Theorem, we proceed by induction (I) on $N \geq 2$. Suppose the Main Theorem is true for $N-1 \geq 2$, and consider an $N$-fold cross $X:=$ $\mathbb{X}\left(A_{1}, \ldots, A_{N} ; D_{1}, \ldots, D_{N}\right)$, where $D_{1}, \ldots, D_{N}$ are pseudoconvex domains and $A_{1}, \ldots, A_{N}$ are open subsets of $\partial D_{1}, \ldots, \partial D_{N}$ such that $D_{j}$ is locally $\mathcal{C}^{2}$ smooth on $A_{j}(1 \leq j \leq N)$. Fix $f \in \mathcal{C}(X) \cap \mathcal{O}_{\mathrm{s}}\left(X^{\mathrm{o}}\right)$.

We next proceed by induction (II) on the positive integer $j(1 \leq j \leq N)$ such that $D_{j}, \ldots, D_{N}$ are Jordan planar domains.

For $j=1$, we are reduced to Theorem 2 .

Suppose the Main Theorem is true if $D_{j-1}, \ldots, D_{N}$ are Jordan planar domains $(j \geq 2)$, and consider the case where $D_{j}, \ldots, D_{N}$ are Jordan planar domains. The proof given below follows essentially the schema of that of Theorem 5.1. It is divided into three steps.

STEP 1: Reduction to the case where $D_{1}, \ldots, D_{j-1}$ are bounded pseudoconvex domains.

Proof of Step 1. We proceed exactly as in Step 1 of Theorem 5.1.

From now on we assume that the hypothesis of Step 1 is fulfilled.

STEP 2: The following local version of the Main Theorem holds:

For any $P \in A_{1}$, there is a ball $\mathcal{A}$ in $A_{1}$ with center $P$ such that for any $f \in \mathcal{C}(X) \cap \mathcal{O}_{\mathrm{s}}\left(X^{\mathrm{o}}\right)$, there is a unique $\widehat{f} \in \mathcal{C}\left(\widehat{X}_{\mathcal{A}}\right) \cap \mathcal{O}\left(\widehat{X}_{\mathcal{A}}^{\mathrm{o}}\right)$ with $\widehat{f}=f$ on $X_{\mathcal{A}}$, where

$$
\begin{aligned}
X_{\mathcal{A}} & :=\mathbb{X}\left(\mathcal{A}, A_{2}, \ldots, A_{N} ; D_{1}, \ldots, D_{N}\right), \\
\widehat{X}_{\mathcal{A}} & :=\widehat{\mathbb{X}}\left(\mathcal{A}, A_{2}, \ldots, A_{N} ; D_{1}, \ldots, D_{N}\right), \\
\widehat{X}_{\mathcal{A}}^{\mathrm{o}} & :=\widehat{\mathbb{X}}^{\mathrm{o}}\left(\mathcal{A}, A_{2}, \ldots, A_{N} ; D_{1}, \ldots, D_{N}\right) .
\end{aligned}
$$

Proof of Step 2. As in Step 2 of the proof of Theorem 5.1 we first apply Proposition 2.5 to the domain $D_{1}$ which is locally $\mathcal{C}^{2}$ smooth on an open neighborhood of $P$ in $\partial D_{1}$. Consequently, there is an open neighborhood $U$ of $P$ satisfying (2.2) such that Proposition 2.5 is applicable there. Again, $U$, $U_{1}, \pi^{\mathbb{C}}, V$ and $V_{Q}$ have the same meaning as in Proposition 2.5. Now we fix a ball $\mathcal{A}:=A_{1} \cap B(P, r)$ with $r$ so small that $2 \mathcal{A} \Subset A_{1}$ etc.

Arguing as in (5.2)-(5.3) and (5.4)-(5.8), we can choose a $\delta_{0}>0$ and a constant $C$ such that for any $0<\delta<\delta_{0}$ there is an open subset $T_{\delta}^{1}$ of $D_{1}$ satisfying

$$
\omega\left(z_{1}, \mathcal{A}, D_{1}\right)-\delta \leq \omega\left(z_{1}, T_{\delta}^{1}, D_{1}\right) \leq \omega\left(z_{1}, \mathcal{A}, D_{1}\right), \quad z_{1} \in D_{1},
$$

$$
\sup _{T_{\delta}^{1}} \operatorname{dist}(\cdot, \mathcal{A})<\delta / C, \quad T_{\delta}^{1} \subset \bigcup_{Q \in \pi^{\mathbb{C}}(2 \mathcal{A})} V_{Q},
$$

and 


$$
\omega\left(z_{1}, \partial V_{Q_{z_{1}}} \cap 2 \mathcal{A}, V_{Q_{z_{1}}}\right) \leq C \operatorname{dist}\left(z_{1}, \mathcal{A}\right) \leq \delta
$$

for $0<\delta<\delta_{0}, z_{1} \in T_{\delta}^{1}$ and $Q_{z_{1}}:=\pi^{\mathbb{C}}\left(z_{1}\right)$.

Similarly, for each $2 \leq k \leq N$ there is an open subset $T_{\delta}^{k}$ of $D_{k}$ satisfying

$$
\omega\left(z_{k}, A_{k}, D_{k}\right)-\delta \leq \omega\left(z_{k}, T_{\delta}^{k}, D_{k}\right) \leq \omega\left(z_{k}, A_{k}, D_{k}\right), \quad z_{k} \in D_{k} .
$$

For each $Q \in \pi^{\mathbb{C}}(2 \mathcal{A})$, we apply the induction assumption (II) to $f \in \mathcal{C}\left(\mathbb{X}\left(2 \mathcal{A} \cap \partial V_{Q}, A_{2}, \ldots, A_{N} ; V_{Q}, D_{2}, \ldots, D_{N}\right)\right)$

$$
\cap \mathcal{O}_{\mathrm{s}}\left(\mathbb{X}^{\mathrm{o}}\left(2 \mathcal{A} \cap \partial V_{Q}, A_{2}, \ldots, A_{N} ; V_{Q}, D_{2}, \ldots, D_{N}\right)\right)
$$

to obtain

$$
\begin{aligned}
\widehat{f}_{Q} \in \mathcal{C}\left(\widehat{\mathbb{X}}\left(2 \mathcal{A} \cap \partial V_{Q}, A_{2}, \ldots, A_{N} ; V_{Q}, D_{2}, \ldots, D_{N}\right)\right) \\
\cap \mathcal{O}\left(\widehat{\mathbb{X}}^{\mathrm{o}}\left(2 \mathcal{A} \cap \partial V_{Q}, A_{2}, \ldots, A_{N} ; V_{Q}, D_{2}, \ldots, D_{N}\right)\right)
\end{aligned}
$$

such that

$$
\widehat{f}_{Q}=f \quad \text { on } \mathbb{X}\left(\mathcal{A} \cap \partial V_{Q}, A_{2}, \ldots, A_{N} ; V_{Q}, D_{2}, \ldots, D_{N}\right) .
$$

Gluing the family $\left(\widehat{f}_{Q}\right)_{Q \in \pi^{\mathbb{C}}(2 \mathcal{A})}$, we obtain an extension function $\widetilde{f}$ defined on

$$
\begin{aligned}
\left\{\left(z_{1}, \ldots, z_{N}\right) \in D_{1} \times\right. & \cdots \times D_{N}: \exists Q \in \pi^{\mathbb{C}}(2 \mathcal{A}), z_{1} \in V_{Q} \text { and } \\
& \left.\omega\left(z_{1}, 2 \mathcal{A} \cap \partial V_{Q}, V_{Q}\right)+\sum_{k=2}^{N} \omega\left(z_{k}, A_{k}, D_{k}\right)<1\right\}
\end{aligned}
$$

which satisfies

$$
\widetilde{f}=f \quad \text { on } \mathbb{X}\left(\mathcal{A}, A_{2}, \ldots, A_{N} ; T_{\delta_{0}}^{1}, D_{2}, \ldots, D_{N}\right) .
$$

For $0 \leq \delta<\delta_{0}$ put

$$
\begin{array}{r}
D_{\delta}^{\prime}:=\left\{\left(z_{2}, \ldots, z_{N}\right) \in D_{2} \times \cdots \times D_{N}: \omega\left(z_{2}, A_{2}, D_{2}\right)+\omega\left(z_{2}, T_{\delta}^{3}, D_{3}\right)\right. \\
\left.+\cdots+\omega\left(z_{N}, T_{\delta}^{N}, D_{N}\right)<1-N \delta\right\}
\end{array}
$$

and

$$
D_{\delta}^{k}:=\left\{z_{k} \in D_{k}: \omega\left(z_{k}, A_{k}, D_{k}\right)<1-N \delta\right\}, \quad 1 \leq k \leq N .
$$

Consequently, in view of (6.1)-(6.4) and (6.6) for any fixed $z_{1} \in T_{\delta}^{1}$ and $0<\delta<\delta_{0}$, the restriction $\widetilde{f}\left(z_{1}, \cdots\right)$ is holomorphic on $D_{\delta}^{\prime}$.

On the other hand, for any $a_{2} \in A_{2}$, by the induction assumption (I) for an $(N-1)$-fold cross, we obtain an extension $\widehat{f}_{a_{2}}$ such that

$$
\begin{aligned}
\widehat{f}_{a_{2}} \in \mathcal{C}\left(\widehat{\mathbb{X}}\left(A_{1}, A_{3}, \ldots, A_{N} ; D_{1}, \ldots, D_{N}\right)\right) \\
\cap \mathcal{O}\left(\widehat{\mathbb{X}}^{\circ}\left(A_{1}, A_{3}, \ldots, A_{N} ; D_{1}, \ldots, D_{N}\right)\right)
\end{aligned}
$$


and

$$
\begin{aligned}
& \widehat{f}_{a_{2}}\left(z_{1}, z_{3}, \ldots, z_{N}\right)=f\left(z_{1}, a_{2}, z_{3}, \ldots, z_{N}\right) \\
& \quad\left(z_{1}, z_{3}, \ldots, z_{N}\right) \in \mathbb{X}\left(A_{1}, A_{3}, \ldots, A_{N} ; D_{1}, D_{3}, \ldots, D_{N}\right) .
\end{aligned}
$$

Observe that by (6.1)-(6.3), (6.6), and (6.9)-(6.10), for $0<\delta<\delta_{0}$ and $\delta_{0}$ sufficiently small, the domain of definition of $\widehat{f}_{a_{2}}\left(a_{2} \in A_{2}\right)$ contains $D_{\delta}^{1} \times T_{\delta}^{3} \times \cdots \times T_{\delta}^{N}$ and that of $\tilde{f}$ contains $T_{\delta}^{1} \times A_{2} \times T_{\delta}^{3} \times \cdots \times T_{\delta}^{N}$. Next we prove that for $0<\delta<\delta_{0}$ with $\delta$ sufficiently small, and $a_{2} \in A_{2}$,

$$
\begin{aligned}
\tilde{f}\left(z_{1}, a_{2}, z_{3}, \ldots, z_{N}\right)=\widehat{f}_{a_{2}}\left(z_{1}, z_{3}, \ldots, z_{N}\right), \\
\left(z_{1}, z_{3}, \ldots, z_{N}\right) \in T_{\delta}^{1} \times T_{\delta}^{3} \times \cdots \times T_{\delta}^{N} .
\end{aligned}
$$

Indeed, by (6.5) and (6.11) and by applying the induction assumption (I) to $\widehat{f}_{a_{2}}$ and the induction assumption (II) to $\widehat{f}_{Q}$ for any $Q \in \pi^{\mathbb{C}}(2 \mathcal{A})$ we know that

$$
\begin{aligned}
\widetilde{f}\left(z_{1}, a_{2}, z_{3}, \ldots, z_{N}\right) & =\widehat{f}_{Q}\left(z_{1}, a_{2}, z_{3}, \ldots, z_{N}\right)=f\left(z_{1}, a_{2}, z_{3}, \ldots, z_{N}\right) \\
& =\widehat{f}_{a_{2}}\left(z_{1}, z_{3}, \ldots, z_{N}\right), \\
z_{1} \in \mathcal{A} \cap \partial V_{Q}, & a_{2} \in A_{2}, \quad\left(z_{3}, \ldots, z_{N}\right) \in \mathbb{X}\left(A_{3}, \ldots, A_{N} ; D_{3}, \ldots, D_{N}\right) .
\end{aligned}
$$

This proves (6.12). Consequently, we can define a new function $\widetilde{f}_{\delta}$ on $\mathbb{X}\left(T_{\delta}^{1}\right.$, $\left.A_{2} \times T_{\delta}^{3} \times \cdots \times T_{\delta}^{N} ; D_{\delta}^{1}, D_{\delta}^{\prime}\right)$ as follows:

$$
\widetilde{f}_{\delta}:= \begin{cases}\widetilde{f} & \text { on } T_{\delta}^{1} \times D_{\delta}^{\prime}, \\ \widehat{f}_{a_{2}} & \text { on } T_{\delta}^{1} \times\left\{a_{2}\right\} \times T_{\delta}^{3} \times \cdots \times T_{\delta}^{N}, a_{2} \in A_{2} .\end{cases}
$$

We need the following lemmas:

LEMMA 6.1.

(1) $\widetilde{f}_{\delta}$ is locally bounded on $\mathbb{X}\left(T_{\delta}^{1}, A_{2} \times T_{\delta}^{3} \times \cdots \times T_{\delta}^{N} ; D_{\delta}^{1}, D_{\delta}^{\prime}\right)$;

(2) $\widetilde{f}_{\delta}$ is locally bounded on $T_{\delta}^{1} \times\left(D_{\delta}^{\prime} \cup\left(\overline{D_{\delta}^{\prime}} \cap \mathbb{X}\left(A_{2}, \ldots, A_{N} ; D_{2}, \ldots, D_{N}\right)\right)\right)$ and $\widetilde{f}_{\delta}\left(z_{1}, \cdots\right) \in \mathcal{C}\left(D_{\delta}^{\prime} \cup\left(\overline{D_{\delta}^{\prime}} \cap \mathbb{X}\left(A_{2}, \ldots, A_{N} ; D_{2}, \ldots, D_{N}\right)\right)\right)$ for any $z_{1} \in T_{\delta}^{1}$.

Proof of Lemma 6.1. It follows the lines of that of Lemma 5.2. Therefore, we only indicate a crucial difference: in Lemma 5.2 we appeal to Gonchar's Theorem, while in the present lemma we apply the induction hypothesis (II).

Lemma 6.2. Let $D_{2}$ be a bounded open set and let $A_{2}$ be an open subset of $\partial D_{2}$ such that $D_{2}$ is locally $\mathcal{C}^{2}$ smooth on $A_{2}$. Let $T^{k} \subset D_{k} \Subset \mathbb{C}^{n_{k}}, D_{k}$ a domain and $T^{k}$ locally pluriregular, $k=3, \ldots, N, N \geq 3$. Put 


$$
\begin{aligned}
& D^{\prime}:=\left\{z^{\prime}=\left(z_{2}, \ldots, z_{N}\right) \in D_{2} \times \cdots \times D_{N}:\right. \\
&\left.\omega\left(z_{2}, A_{2}, D_{2}\right)+\sum_{k=3}^{N} \omega\left(z_{k}, T^{k}, D_{k}\right)<1\right\}
\end{aligned}
$$

Then

$$
\omega\left(z^{\prime}, A_{2} \times T^{3} \times \cdots \times T^{N}, D^{\prime}\right)=\omega\left(z_{2}, A_{2}, D_{2}\right)+\sum_{k=3}^{N} \omega\left(z_{k}, T^{k}, D_{k}\right) .
$$

Proof of Lemma 6.2. We argue as in the proof of Lemma 3(b) in [7] making use of Proposition 3.8(1).

We now come back to the proof of the Main Theorem. Applying Lemma 6.2 and Proposition 3.8(1), we see that

$$
\begin{aligned}
\omega\left(z^{\prime}, A_{2} \times T_{\delta}^{3} \times \cdots \times\right. & \left.T_{\delta}^{N}, D_{\delta}^{\prime}\right) \\
& =\frac{1}{1-N \delta}\left(\omega\left(z_{2}, A_{2}, D_{2}\right)+\sum_{k=3}^{N} \omega\left(z_{k}, T_{\delta}^{k}, D_{k}\right)\right)
\end{aligned}
$$

for any $z^{\prime} \in D_{\delta}^{\prime}$.

To summarize what has been done so far: for any $0<\delta<\delta_{0}$ sufficiently small, we obtain, by Lemma $6.1(1)$, a function $\widetilde{f}_{\delta}$ defined on a mixed cross,

$$
\begin{aligned}
\tilde{f}_{\delta} \in \mathcal{C}_{\mathrm{S}}\left(\mathbb { X } \left(T_{\delta}^{1}, A_{2} \times T_{\delta}^{3} \times\right.\right. & \left.\left.\cdots \times T_{\delta}^{N} ; D_{\delta}^{1}, D_{\delta}^{\prime}\right)\right) \\
& \cap \mathcal{O}_{s}\left(\mathbb{X}^{\mathrm{o}}\left(T_{\delta}^{1}, A_{2} \times T_{\delta}^{3} \times \cdots \times T_{\delta}^{N} ; D_{\delta}^{1}, D_{\delta}^{\prime}\right)\right) .
\end{aligned}
$$

Applying Theorem 4.1 to $\widetilde{f}_{\delta}$ we obtain an extension function $\widetilde{\widetilde{f}}_{\delta}$ of $\widetilde{f}_{\delta}$ such that

$$
\begin{aligned}
\widetilde{\widetilde{f}}_{\delta} \in \mathcal{C}\left(\widehat{\mathbb{X}}\left(T_{\delta}^{1}, A_{2} \times T_{\delta}^{3} \times \cdots \times T_{\delta}^{N} ; D_{\delta}^{1}, D_{\delta}^{\prime}\right)\right) \\
\\
\cap \mathcal{O}\left(\widehat{\mathbb{X}}^{\mathrm{O}}\left(T_{\delta}^{1}, A_{2} \times T_{\delta}^{3} \times \cdots \times T_{\delta}^{N} ; D_{\delta}^{1}, D_{\delta}^{\prime}\right)\right) .
\end{aligned}
$$

In view of (6.4)-(6.9) and (6.13)-(6.15) and by Lemma 6.1(2), we can apply Theorem $4.1(2)$ to conclude that $\widetilde{\widetilde{f}}_{\delta}$ can be continuously extended to

$$
\widehat{f}_{\delta} \in \mathcal{C}\left(\widehat{X}_{\delta}\right)
$$

where

$$
\cup\left(\widehat{\widehat{\mathbb{X}}}\left(T_{\delta}^{1}, A_{2} \times T_{\delta}^{3} \times \cdots \times T_{\delta}^{N} ; D_{\delta}^{1}, D_{\delta}^{\prime}\right) \cap\left(D_{\delta}^{1} \times \mathbb{X}\left(A_{2}, \ldots, A_{N} ; D_{2}, \ldots, D_{N}\right)\right)\right) .
$$

We are now in a position to define the desired extension function $\widehat{f}$. Indeed, one glues $\left(\widehat{f}_{\delta}\right)_{0<\delta<\delta_{0}}$ together to obtain $\widehat{f}$ in the following way:

$$
\widehat{f}:=\lim _{\delta \rightarrow 0} \widehat{f}_{\delta} \quad \text { on } \widehat{X}_{\mathcal{A}} \backslash\left(\mathcal{A} \times \mathbb{X}\left(A_{2}, \ldots, A_{N} ; D_{2}, \ldots, D_{N}\right)\right) .
$$


One has to check that the limit exists and has all the required properties. This is an immediate consequence of

Lemma 6.3. For any

$$
z=\left(z_{1}, \ldots, z_{N}\right) \in \widehat{X}_{\mathcal{A}} \backslash\left(\mathcal{A} \times \mathbb{X}\left(A_{2}, \ldots, A_{N} ; D_{2}, \ldots, D_{N}\right)\right)
$$

let $\delta_{z}$ be the unique positive number $\delta$ which satisfies

$$
\delta+\frac{2 N \delta}{1-N \delta}=1-\omega\left(z_{1}, \mathcal{A}, D_{1}\right)-\sum_{k=2}^{N} \omega\left(z_{k}, A_{k}, D_{k}\right) .
$$

Then $f(z)=\widehat{f}_{\delta}(z)$ for all $0<\delta<\delta_{z}$.

Proof of Lemma 6.3. Fix $z^{0}=\left(z_{1}^{0}, \ldots, z_{N}^{0}\right) \in \widehat{X}_{\mathcal{A}} \backslash\left(\mathcal{A} \times \mathbb{X}\left(A_{2}, \ldots, A_{N} ;\right.\right.$ $\left.\left.D_{2}, \ldots, D_{N}\right)\right)$. Then by $(6.1)-(6.3)$ and $(6.14)-(6.18)$ and the second identity of Proposition 3.8(1), for all $0<\delta<\delta_{z^{0}}$, we have $z^{0} \in \widehat{X}_{\delta}$. In particular, $\bigcup_{0<\delta<\delta_{0}} \widehat{X}_{\delta}=\widehat{X}_{\mathcal{A}} \backslash\left(\mathcal{A} \times \mathbb{X}\left(A_{2}, \ldots, A_{N} ; D_{2}, \ldots, D_{N}\right)\right)$.

Therefore, for any $0<\delta^{\prime}<\delta<\delta_{z^{0}}, z^{0}$ is in the set

$$
\widehat{X}_{\delta} \cap \widehat{X}_{\delta^{\prime}} \text {. }
$$

Let $H_{\delta}$ be the connected component of $D_{\delta}^{\prime}$ containing $\left(z_{2}^{0}, \ldots, z_{n}^{0}\right)$. Let $B_{\delta}$ be the largest open subset of $B$ such that $H_{\delta}$ is locally $\mathcal{C}^{2}$ smooth on $B_{\delta}$. By (6.1)-(6.3) and (6.19) and arguing as in the proof of Lemma 5.3, we see that the above intersection contains

$$
\left\{z=\left(z_{1}, z^{\prime}\right) \in D_{\delta}^{1} \times H_{\delta}: \omega\left(z_{1}, T_{\delta}^{1}, D_{\delta}^{1}\right)+\omega\left(z^{\prime}, B_{\delta}, H_{\delta}\right)<1-\delta\right\} .
$$

By Proposition 3.7 this open set is connected. Moreover, it contains $z^{0}$. In addition we deduce from (6.17) that

$$
\widehat{f_{\delta^{\prime}}}=\widehat{f_{\delta}}=\widetilde{f}=f \quad \text { on } T_{\delta^{\prime}}^{1} \times B_{\delta^{\prime}} .
$$

Theorem 2.1 shows that $\widehat{f}_{\delta^{\prime}}=\widehat{f}_{\delta}$ on the domain given by (6.20). In particular $\widehat{f}_{\delta^{\prime}}\left(z^{0}\right)=\widehat{f}_{\delta}\left(z^{0}\right)$. This completes the proof.

An immediate consequence of Lemma 6.3 is that $\widehat{f} \in \mathcal{O}\left(\widehat{X}_{\mathcal{A}}^{\mathrm{o}}\right)$. Now we define $\widehat{f}$ to be $f$ on $\mathcal{A} \times \mathbb{X}\left(A_{2}, \ldots, A_{N} ; D_{2}, \ldots, D_{N}\right)$. Thus $\widehat{f}$ is well defined on the whole $\widehat{X}_{\mathcal{A}}$ and

$$
\widehat{f} \in \mathcal{C}\left(D_{1} \times \mathbb{X}\left(A_{2}, \ldots, A_{N} ; D_{2}, \ldots, D_{N}\right)\right) .
$$

To complete Step 2, it remains to show that $\widehat{f} \in \mathcal{C}\left(\widehat{X}_{\mathcal{A}}\right)$ and $\widehat{f}=f$ on $X_{\mathcal{A}}$. For this purpose we do the following trick.

We replace $D_{1}$ by $D_{j}(j=2, \ldots, N)$ and proceed as above. For example, if we replace $D_{1}$ by $D_{2}$, then we obtain a new extension function $\widehat{\hat{f}}$ such that by (6.21),

$$
\widehat{\hat{f}} \in \mathcal{C}\left(D_{2} \times \mathbb{X}\left(A_{1}, A_{3} \ldots, A_{N} ; D_{1}, D_{3}, \ldots, D_{N}\right)\right) .
$$


Next, using identities (6.12), (6.13), (6.16) and (6.18) and applying Theorem 2 , we see that the values of $\widehat{f}$ and $\widehat{\hat{f}}$ can be uniquely determined on $T_{\delta}^{1} \times T_{\delta}^{2} \times \cdots \times T_{\delta}^{N}$ from the value of $f$ on $\mathcal{A} \times A_{2} \times \cdots \times A_{N}$ for any sufficiently small $\delta>0$. Thus

$$
\widehat{\hat{f}}=\widehat{f} \quad \text { on } T_{\delta}^{1} \times T_{\delta}^{2} \times \cdots \times T_{\delta}^{N} .
$$

Hence $\widehat{\hat{f}}=\widehat{f}$ on $\widehat{X}_{\mathcal{A}}$ since $\widehat{X}_{\mathcal{A}}$ is a domain by Proposition 3.6. Therefore, in view of $(6.21),(6.22)$ and similar conclusions with $D_{1}$ replaced by $D_{3}, \ldots, D_{N}$, we conclude that

$$
\widehat{f} \in \mathcal{C}\left(\widehat{X}_{\mathcal{A}} \backslash\left(\mathcal{A} \times A_{2} \times \cdots \times A_{N}\right)\right) .
$$

Therefore, Step 2 will be finished if we can prove that $\widehat{f}$ is continuous on $\mathcal{A} \times A_{2} \times \cdots \times A_{N}$.

To do this fix $a=\left(a_{1}, \ldots, a_{N}\right) \in \mathcal{A} \times A_{2} \times \cdots \times A_{N}$ and $\varepsilon>0$. Next, we apply Proposition 2.5 to each domain $D_{j}$ which is locally $\mathcal{C}^{2}$ smooth on an open neighborhood of $a_{j}, j=1, \ldots, N$. Consequently, there is an open neighborhood $U^{j}$ of $a_{j}$ satisfying (2.2) such that Proposition 2.5 is applicable there. Below, $U^{j}, U_{1}^{j}, \pi^{\mathbb{C}, j}, V^{j}$ and $V_{Q}^{j}$ have the same meaning for $a_{j}$ as have $U, U_{1}, \pi^{\mathbb{C}}, V$ and $V_{Q}$ for $P$ in Proposition 2.5.

Since $f \in \mathcal{C}(X)$, by shrinking $U^{j}$ if necessary, we may assume without loss of generality that

$$
\begin{aligned}
& |f(\zeta)-f(\eta)|<\varepsilon / 2, \\
& \quad \zeta, \eta \in \mathbb{X}\left(\mathcal{A} \cap U^{1}, A_{2} \cap U^{2}, \ldots, A_{N} \cap U^{N} ; D_{1} \cap U^{1}, \ldots, D_{N} \cap U^{N}\right) .
\end{aligned}
$$

Let $z=\left(z_{1}, \ldots, z_{N}\right) \in U^{1} \times \cdots \times U^{N}$ and put $Q_{j}:=\pi^{\mathbb{C}, j}\left(z_{j}\right)$. Then, by the hypothesis on $f$, we may apply Theorem 2 to

$$
\begin{aligned}
& f \in \mathcal{C}\left(\mathbb{X}\left(\mathcal{A} \cap \partial V_{Q_{1}}^{1}, A_{2} \cap \partial V_{Q_{2}}^{2}, \ldots, A_{N} \cap \partial V_{Q_{N}}^{N} ; V_{Q_{1}}^{1}, \ldots, V_{Q_{N}}^{N}\right)\right) \\
& \cap \mathcal{O}\left(\mathbb{X}^{\circ}\left(\mathcal{A} \cap \partial V_{Q_{1}}^{1}, A_{2} \cap \partial V_{Q_{2}}^{2}, \ldots, A_{N} \cap \partial V_{Q_{N}}^{N} ; V_{Q_{1}}^{1}, \ldots, V_{Q_{N}}^{N}\right)\right) .
\end{aligned}
$$

Consequently, taking into account (6.23) and the above construction of $\widehat{f}$, we deduce that

$$
\begin{aligned}
& |\widehat{f}(z)-f(\zeta)|<\varepsilon / 2, \\
& \quad \quad \quad \in \mathbb{X}\left(\mathcal{A} \cap \partial V_{Q_{1}}^{1}, A_{2} \cap \partial V_{Q_{2}}^{2}, \ldots, A_{N} \cap \partial V_{Q_{N}}^{N} ; V_{Q_{1}}^{1}, \ldots, V_{Q_{N}}^{N}\right) .
\end{aligned}
$$

Hence, fixing any $\zeta$ as above and applying (6.23) again, we get

$$
|\widehat{f}(z)-f(a)| \leq|\widehat{f}(z)-f(\zeta)|+|f(\zeta)-f(a)|<\varepsilon / 2+\varepsilon / 2<\varepsilon,
$$

which proves the continuity of $\widehat{f}$ at $a$. Hence the remaining assertion of Step 2 is proved.

Thus the inductive proofs (I) and (II) are complete in this second step.

STEP 3: The general case. 
The argument used to go from Step 2 to Step 3 in the proof of Theorem 5 also enables us to go from Step 2 to Step 3 in the present context. Consequently, there is an $\widehat{f} \in \mathcal{C}(\widehat{X}) \cap \mathcal{O}_{\mathrm{s}}\left(\widehat{X}^{\mathrm{o}}\right)$ such that $\widehat{f}=f$ on $X$. It is also clear that $\widehat{f}$ is uniquely determined. Finally, it remains to establish estimate (1.3). We have already proved the existence and uniqueness of the Main Theorem. Using this result we argue as in the proof of Theorem 4.2 to obtain (1.3). This completes the last step of the proof.

Hence the Main Theorem is proved.

We conclude this paper with a remark and an open question.

1. It seems to be of interest to establish the Main Theorem under weaker assumptions than the continuity of $f$, the smoothness of $D_{j}$ on $A_{j}$, the regularity of the set $A_{j}, j=1, \ldots, N$, etc. We postpone this issue to an ongoing work.

2. Does the Main Theorem still hold if we only assume that $A_{j}$ is of positive $\left(2 n_{j}-1\right)$-Hausdorff measure, $j=1, \ldots, N$ ?

\section{References}

[1] O. Alehyane et A. Zeriahi, Une nouvelle version du théorème d'extension de Hartogs pour les applications séparément holomorphes entre espaces analytiques, Ann. Polon. Math. 76 (2001), 245-278.

[2] L. M. Drużkowski, A generalization of the Malgrange-Zerner theorem, ibid. 38 (1980), 181-186.

[3] A. A. Gonchar, On Bogolyubov's "edge of the wedge" theorem, Proc. Steklov Inst. Math. 228 (2000), 18-24.

[4] -, On analytic continuation from the "edge of the wedge" theorem, Ann. Acad. Sci. Fenn. Ser. A.I. Math. 10 (1985), 221-225.

[5] G. M. Henkin and E. M. Chirka, Boundary properties of holomorphic functions of several complex variables, J. Soviet Math. 5 (1976), 612-687.

[6] M. Jarnicki and P. Pflug, Extension of Holomorphic Functions, de Gruyter Exp. Math. 34, de Gruyter 2000.

[7] - - - An extension theorem for separately holomorphic functions with analytic singularities, Ann. Polon. Math. 80 (2003), 143-161.

[8] N. Kerzman, Topics in Complex Analysis, unpublished notes.

[9] M. Klimek, Pluripotential Theory, London Math. Soc. Monogr. 6, Oxford Univ. Press, 1991.

[10] H. Komatsu, A local version of Bochner's tube theorem, J. Fac. Sci. Univ. Tokyo Sect. I A 19 (1972), 201-214.

[11] S. G. Krantz, Function Theory of Several Complex Variables, 2nd ed., Wadsworth Brooks/Cole Advanced Books \& Software, Pacific Grove, CA, 1992.

[12] Nguyên Thanh Vân et J. Siciak, Fonctions plurisousharmoniques extrémales et systèmes doublement orthogonaux de fonctions analytiques, Bull. Sci. Math. 115 (1991), 235-244. 
[13] Nguyên Thanh Vân et A. Zeriahi, Une extension du théorème de Hartogs sur les fonctions séparément analytiques, in: Analyse Complexe Multivariable, Récents Développements, A. Meril (ed.), EditEl, Rende, 1991, 183-194.

[14] P. Pflug, Extension of separately holomorphic functions - a survey 1899-2001, Ann. Polon. Math. 80 (2003), 21-36.

[15] T. Ransford, Potential Theory in the Complex Plane, London Math. Soc. Student Texts 28, Cambridge Univ. Press, 1995.

[16] A. Sadullaev, Plurisubharmonic measures and capacities on complex manifolds, Russian Math. Surveys 36 (1981), 61-119.

[17] M. Zerner, Quelques résultats sur le prolongement analytique des fonctions de variables complexes, Séminaire de Physique Mathématique.

Fachbereich Mathematik

Carl von Ossietzky Universität Oldenburg

Postfach 2503

D-26111 Oldenburg, Germany

E-mail: pflug@mathematik.uni-oldenburg.de nguyen@mathematik.uni-oldenburg.de 